\title{
Characterization of mixing and spreading in a bounded stratified medium
}

\author{
Vanessa Zavala-Sanchez, Marco Dentz*, Xavier Sanchez-Vila \\ Department of Geotechnical Engineering and Geosciences, Technical University of Catalonia (UPC), Campus Nord, Modulo D 2, 8034 Barcelona, Spain
}

\section{A R T I C L E I N F O}

\section{Article history:}

Received 21 November 2007

Received in revised form 19 May 2008

Accepted 26 May 2008

Available online 14 July 2008

\section{Keywords:}

Contaminant transport

Stratified medium

Taylor problem

Stochastic modeling

Mixing and spreading

Dispersion

Mixing dynamics

Superdiffusion

\begin{abstract}
A B S T R A C T
Matheron and de Marsily [Matheron M, de Marsily G. Is the transport in porous media always diffusive? A counter-example. Water Resour Res 1980;16:901-17] studied transport in a perfectly stratified infinite medium as an idealized aquifer model. They observed superdiffusive solute spreading quantified by anomalous increase of the apparent longitudinal dispersion coefficient with the square root of time. Here, we investigate solute transport in a vertically bounded stratified random medium. Unlike for the infinite medium at asymptotically long times, disorder-induced mixing and spreading is uniquely quantified by a constant Taylor dispersion coefficient. Using a stochastic modeling approach we study the effective mixing and spreading dynamics at pre-asymptotic times in terms of effective average transport coefficients. The latter are defined on the basis of local moments, i.e., moments of the transport Green function. We investigate the impact of the position of the initial plume and the initial plume size on the (highly anomalous) pre-asymptotic effective spreading and mixing dynamics for single realizations and in average. Effectively, the system "remembers" its initial state, the effective transport coefficients show so-called memory effects, which disappear after the solute has sampled the full vertical extent of the medium. We study the impact of the intrinsic non-ergodicity of the confined medium on the validity of the stochastic modeling approach and study in this context the transition from the finite to the infinite medium. (c) 2008 Elsevier Ltd. All rights reserved.
\end{abstract}

\section{Introduction}

Transport in stratified media has been frequently studied in the groundwater literature as a model for transport in geological media. Natural sandy aquifers often exhibit geological and geostatistical stratification characterized by a much larger horizontal than vertical correlation length (see, e.g. [2] and literature therein). In the limiting case of infinite correlation length in the horizontal direction, the hydraulic conductivity varies only along the vertical. Following the deterministic work of Marle et al. [3], Matheron and de Marsily [1] studied this perfectly stratified medium as an idealized aquifer model. They found that the apparent longitudinal dispersion coefficient grows superdiffusively with the square root of time and used this result to demonstrate that transport in porous media is not always diffusive. Transport in an infinite perfectly stratified random medium has been investigated extensively (e.g. [4-11]) using stochastic modeling as a systematic means to quantify the impact of spatial heterogeneity on large scale transport. The latter has been studied in terms of the average solute distribution density and its moments, its spatial and temporal moments as well as in terms of (apparent) longitudinal dispersion coefficients.

The superdiffusive growth of the apparent longitudinal dispersion coefficient is caused by strong spatial correlation as quantified

\footnotetext{
* Corresponding author. Fax: +34 934017251.

E-mail address: marco.dentz@upc.es (M. Dentz).
}

by the Lagrangian velocity correlation (e.g. $[12,13])$. These aspects of transport in stratified flows have been extensively studied in the physics literature (e.g. [14-17]).

In contrast to the unbounded stratified medium, for which transport is superdiffusive for all times, for a vertically (i.e., transverse to the direction of stratification) bounded medium, transport becomes eventually Gaussian and can be completely characterized by a constant macrodispersion or "Taylor dispersion-type" coefficient. Several authors have addressed the issue of enhanced dispersion and effective transport dynamics for bounded stratified random media and shear flows in general (e.g. [2,18-21]). Taylor [22] was the first to quantify enhanced solute dispersion in the parabolic (stratified) Hagen-Poiseuille flow through a tube by the well known Taylor dispersion coefficient

$D^{*} \propto \frac{a^{2} U^{2}}{D_{\mathrm{T}}}$,

where $a$ is a measure for the vertical extent of the flow domain, $U$ the average flow velocity and $D_{\mathrm{T}}$ the transverse local dispersion coefficient, i.e. the transverse component of the (constant) dispersion tensor at local scale. The Taylor dispersion coefficient (1) reflects the mechanism that leads to enhanced spreading and mixing in stratified flows, namely, the solute's sampling of the vertical velocity contrast $\left(U^{2}\right)$ by local transverse dispersion $\left(D_{\mathrm{T}}\right)$. The process is controlled by the dispersion time scale $\tau_{\mathrm{D}}$ 
$\tau_{\mathrm{D}}=\frac{a^{2}}{D_{\mathrm{T}}}$

which measures the time for the solute to sample the whole vertical velocity contrast. For times large compared to the dispersion time scale, $t \gg \tau_{\mathrm{D}}$, the Taylor dispersion coefficient $D^{*}$ quantifies both large scale spreading and mixing as well as the evolution of the solute concentration.

In hydrological applications, however, this dispersion time scale can be large (of the order of $10^{3}$ years). At the relevant pre-asymptotic times, the constant Taylor or macrodispersion coefficient overestimates actual solute spreading and mixing. For risk assessment studies that focus on the maximum extent of a contaminant plume, macrodispersion gives simulates a worst case scenario and maybe the observable of choice. If one is interested in remediation strategies relying on the mixing of contaminated water with an injected reactant, the correct quantification of the pre-asymptotic mixing mechnisms is mandatory in order to be able to realistically assess the efficiency of the remediation strategy. Macrodispersion simulates to high a mixing efficiency and can significantly overestimate the performance of a remediation strategy.

At pre-asymptotic times, i.e., for times smaller than $\tau_{\mathrm{D}}$, solute spreading and mixing is controlled by local transverse dispersion, which activates the vertical velocity contrast as a macroscopic spreading and mixing mechanisms. As outlined in [23], transverse dispersion mixes the solute vertically. The velocity contrast experienced by the solute through vertical mixing stretches the plume and increases the plume surface but not the solute, which is termed spreading then again leads to vertical mass exchang ers and smoothes concentration contr

\section{scale mixing.}

e investigate these mechanisms for a stratified random medium in terms of suitably defined second centered moments of the solute plume. This analysis is based on the moments of

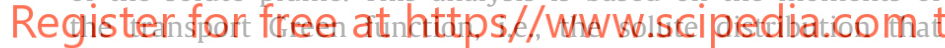
evolves from a point-like initial distribution. The latter allows for the construction of observables that measure spreading and/or mixing of the solute. Many studies of solute dispersion in single realization focus on the vertically averaged (over the directions perpendicular to the direction of stratification) solute concentration and in stochastic frameworks on ensemble averaged concentrations. The vertically averaged solute concentration quantifies (advective) solute spreading within the initial plume [24], the ensemble averaged concentration quantifies an artificial spreading effect due to sample to sample fluctuations of the plume's center of mass from realization to realization [25]. While for transport in heterogeneous media these fluctuations vanish in average for time large compared to the dispersion scale [26], for an infinite stratifies random medium they persist [10]. Furthermore, averaging over a large initial plume or stochastic averaging wipes out possible memory effects that account for the impact of the initial position or initial plume size on the effective transport behavior. We study these mechanisms systematically for single realizations and in stochastic average for confined stratified media using explicit analytical expressions and numerical random walk simulations. We discuss the stochastic approach for such confined scenarios and the impact of finite size effects on the ergodicity of transport.

In Section 2, we present the specific aquifer model under consideration, which is characterized by a linear covariance function for the conductivity in vertical direction. Section 3 introduces the concepts and defines the transport coefficients used to investigate the different mechanisms described above. This section presents analytical and numerical solution methods axial moment equations and random walk simulations, respectively. We derive expli- cite analytical solutions for the ensemble averaged transport coefficients. Section 4 applies these concepts and methods for the systematic analysis of mixing and spreading, Section 5 concludes the paper.

\section{Model}

We study transport of a conservative solute in a confined horizontally stratified medium. The $d$-dimensional flow and transport domain, denoted by $\Omega^{d}$, is assumed to be of infinite extension at least in the one-direction and finite only in one of the transverse directions.

\subsection{Flow and transport in stratified media}

Flow through a stratified porous medium is characterized by the Darcy equation (e.g. [27])

$\mathbf{u}(\mathbf{x})=-\frac{K(\mathbf{y})}{\phi} \nabla h(\mathbf{x})$

where $\mathbf{u}(\mathbf{x})$ is the pore velocity, $\phi$ is the constant porosity, $\mathbf{x}$ is the position vector in $\Omega^{d}$ and $\mathbf{y}=\left(x_{2}, \ldots, x_{d}\right)^{\mathrm{T}}$ is the position vector in the $(d-1)$-dimensional subdomain $\Omega$, with $\Omega^{d}=\Omega \times \mathbf{R}$. In the following, for simplicity constant porosity is set to 1 . The hydraulic conductivity is denoted by $K(\mathbf{y})$ and varies only in $\Omega, h(\mathbf{x})$ is the hydraulic head. The flow is driven by a constant head gradient $\mathbf{J}$, which is aligned with the direction of stratification, $J=-J \mathbf{e}_{1}$, where

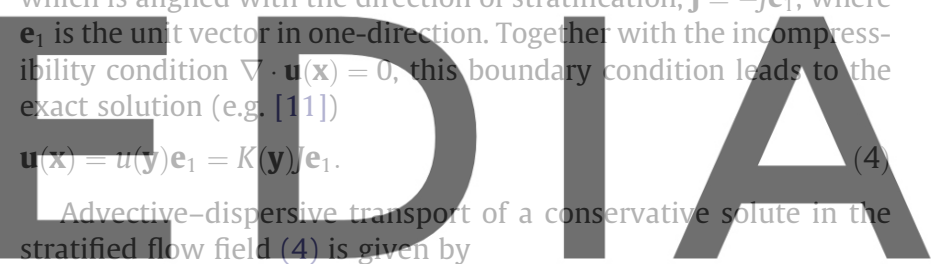

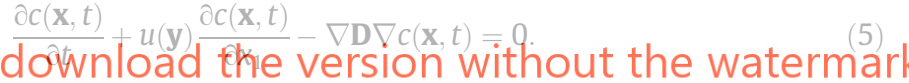

The (constant) local dispersion tensor $\mathbf{D}$ is assumed to be diagonal, $D_{i j}=\delta_{i j} D_{i j}$ with $D_{11}=D_{L}$ and $D_{i i}=D_{\mathrm{T}}$ for $i>1$.

As initial condition, we consider an instantaneous solute injection at time $t=0$

$c(\mathbf{x}, t=0)=\rho(\mathbf{x})$,

where the initial distribution $\rho(\mathbf{x})$ is normalized to one. As the stratified flow velocity is divergence-free, this normalization is conserved for all times. We study transport of a solute evolving from a point-like injection and from an extended source perpendicular to stratification. Both initial conditions will be described in the following. The boundary conditions for $c(\mathbf{x}, t)$ are

$\lim _{x_{1} \rightarrow \pm \infty} c(\mathbf{x}, t)=0,\left.\quad \mathbf{n} \cdot \nabla c(\mathbf{x}, t)\right|_{\mathbf{x} \in \partial \Omega^{d}}=0$,

where $\partial \Omega^{d}$ is the boundary of the transport domain $\Omega^{d}$, and $\mathbf{n}$ is the outward pointing unit vector perpendicular to the domain boundaries.

According to the Duhamel principle, the concentration distribution $c(\mathbf{x}, t)$ can be written as

$c(\mathbf{x}, t)=\int_{\Omega^{d}} \mathrm{~d} \mathbf{x}^{\prime} \rho\left(\mathbf{x}^{\prime}\right) g\left(\mathbf{x}, t \mid \mathbf{x}^{\prime}, 0\right)$,

where the Green function $g\left(\mathbf{x}, t \mid \mathbf{x}^{\prime}, t^{\prime}\right)$ solves the advection-dispersion equation (5) for $\rho(\mathbf{x})=\delta\left(\mathbf{x}-\mathbf{x}^{\prime}\right)$ and the boundary conditions (7).

\subsection{Stochastic model}

We use a stochastic modeling approach to account for the impact of spatial heterogeneity on the effective large scale transport 
behavior. In this approach, a given medium is seen as a typical realization of an ensemble of aquifers.

The hydraulic conductivity $K(\mathbf{y})$ here is modeled as a stationary spatial random field. It can be decomposed into its (constant) mean value $\overline{K(\mathbf{y})}=\bar{K}$ and fluctuations about it

$K(\mathbf{y})=\bar{K}[1-k(\mathbf{y})]$.

where $k(\mathbf{y})$ are the normalized conductivity fluctuations, whose mean is zero by definition. The overbar denotes the ensemble average. The translation invariant autocorrelation function of $k(\mathbf{y})$ is given by

$\overline{k(\mathbf{y}) k\left(\mathbf{y}^{\prime}\right)} \equiv \sigma^{2} C\left(\mathbf{y}-\mathbf{y}^{\prime}\right)$.

where the variance $\sigma^{2}=\overline{k(\mathbf{y})^{2}}$. In the following, the correlation function is assumed to be of short-range, i.e., to decay quickly on the correlation scale $l$.

The stochastic approach to quantify the impact of heterogeneity on effective transport in single realizations is certainly meaningful for infinite media, where ergodicity holds, i.e., the statistical characteristics of the ensemble are reflected in a single medium realization. Thus, here we assume here that the lateral halfwidth $a$ of the medium domain is assumed much larger than the correlation scale $l$. Note, however that the correlation function for the confined medium is not translation invariant, i.e.,

$\overline{k(\mathbf{y}) k\left(\mathbf{y}^{\prime}\right)}=\sigma^{2} C_{\Omega}\left(\mathbf{y}-\mathbf{y}^{\prime} \mid \mathbf{y}^{\prime}\right)$

with $\mathbf{y}, \mathbf{y}^{\prime} \in \Omega$. The $C_{\Omega}\left(\xi \mid \mathbf{y}^{\prime}\right)$ is given by

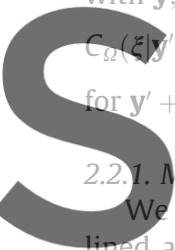
$=C(\xi)$ $\Omega$ and zero elsewhere. Model medium consider here a $d=2$-dime bove the lateral halfwidth
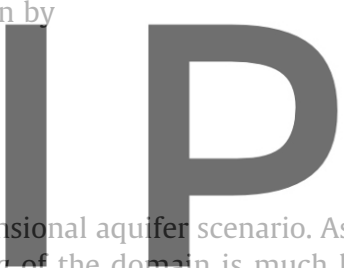

12

than the correlation scale I. Thus, the conductivities in the different

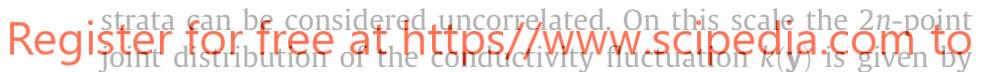
(e.g. [28])

$P_{n}\left(\left\{K^{\prime}\left(\mathbf{y}_{i}\right)\right\}_{i=-n}^{n}\right)=\exp \left\{-\ln P_{1}\left[k^{\prime}\left(\mathbf{y}_{i}\right)\right]\right\}$,

where $P_{1}\left(k^{\prime}\right)$ is the single variable distribution.

The model medium consists of strata of constant thickness $l$, see

Fig. 1. One particular realization of $k(y)$ is given by

$k(y)=\sum_{n=-a / l}^{a / l} k_{n} \Theta[y+b-n l]\{1-\Theta[y+b-(n+1) l]\}$,

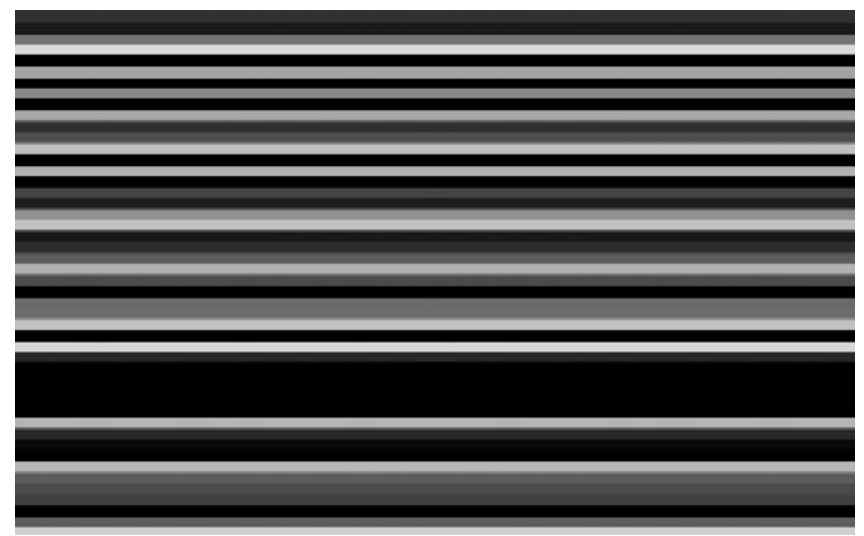

Fig. 1. One realization of a confined stratified medium with 100 strata. Hydraulic conductivity is lognormally distributed; different greyscales denote different values of hydraulic conductivity. where the $k_{n}$ are distributed according to $P_{1}(k), b$ is uniformly distributed in the interval $[-l, l]$, with $\Theta[y+b]$ the Heaviside step function defined in [29]; the position vector is $\mathbf{x}=(x, y)^{\mathrm{T}}$.

This kind of medium is characterized by a linear correlation function (e.g. [30])

$C\left(y-y^{\prime}\right)=l\left\{\frac{1}{2 l}\left[1-\frac{\left|y-y^{\prime}\right|}{l}\right]\right\}$

for $\left|y-y^{\prime}\right|<l$ and 0 elsewhere. It is translation invariant everywhere in the medium except for positions in the strata at the domain boundaries.

If the width $l$ of the strata is small compared to the observation length $L$, i.e. $l / L \ll 1$, (15) can be identified with the delta distribution

$C\left(y-y^{\prime}\right)=l \delta\left(y-y^{\prime}\right)$.

In this limit boundary effects on the correlation function can be disregarded.

\subsubsection{Finite size effects}

Nevertheless, note that the confined stratified medium under consideration here has only a finite number of strata. As such, spatial averages are not necessarily identical to the ensemble averages and are random functions by themselves due to the finiteness of the sample. This can be illustrated for the spatial mean of $k(y)$ for the confined stratified medium

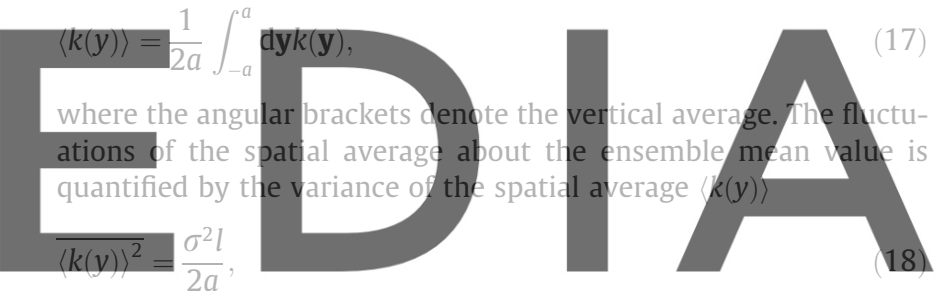

where we used representation (14) for $k(y)$. As expected the vari-

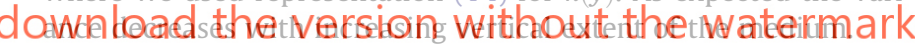

The conductivity field $K(y)$ here is lognormally distributed so that the $k_{n}$ in (14) are given by

$k_{n}=1-\exp \left(f_{n}-\frac{\sigma_{\mathrm{ff}}^{2}}{2}\right)$,

where the $f_{n}$ are normally distributed random variables with zero mean and variance $\sigma_{\mathrm{ff}}^{2}$.

In the following, we use the stochastic approach as a systematic tool to quantify the heterogeneity impact on large scale mixing and spreading.

\subsection{Dimensionless form of the governing equations}

According to (4), the stratified flow field $u(\mathbf{y})$ can be decomposed into its constant mean value and fluctuations about it

$u(\mathbf{y})=\bar{u}-u^{\prime}(\mathbf{y})$,

where mean and fluctuations are given by

$\bar{u}=J \bar{K}, \quad u^{\prime}(\mathbf{y})=\bar{u} k\left(\mathbf{y}^{\prime}\right)$. by

The velocity autocorrelation function is given in terms of $C(\mathbf{y})$

$\overline{u^{\prime}(\mathbf{y}) u^{\prime}\left(\mathbf{y}^{\prime}\right)}=\bar{u}^{2} \sigma^{2} C\left(\mathbf{y}-\mathbf{y}^{\prime}\right)$.

Using decomposition (20) in (5), we obtain

$\frac{\partial c(\mathbf{x}, t)}{\partial t}+\bar{u} \frac{\partial c(\mathbf{x}, t)}{\partial x_{1}}-\nabla \mathbf{D} \nabla c(\mathbf{x}, t)=u^{\prime}(\mathbf{y}) \frac{\partial}{\partial x_{1}} c(\mathbf{x}, t)$,

which is the governing transport equation. 
The observation length $L$, and the flow velocity $\bar{u}$, define the advection time scale $\tau_{u}$

$\tau_{u}=\frac{L}{\bar{u}}$,

which denotes the mean transport time over the distance $L$ along the direction of stratification by mean advection.

We define now non-dimensional time $\hat{t}$ and distance $\hat{x}$ by

$t=\hat{t} \tau_{u}, \quad \mathbf{x}=\hat{\mathbf{x}} L$

respectively. Thus, (23) can be rewritten in non-dimensional terms as

$\frac{\partial \hat{c}(\hat{\mathbf{x}}, \hat{t})}{\partial \hat{t}}+\frac{\partial \hat{c}(\hat{\mathbf{x}}, \hat{t})}{\partial \hat{x}_{1}}-\hat{\nabla} \hat{\mathbf{D}} \hat{\nabla} \hat{c}(\hat{\mathbf{x}}, \hat{t})=\hat{k}(\hat{\mathbf{y}}) \frac{\partial}{\partial \hat{x}_{1}} \hat{c}(\hat{\mathbf{x}}, \hat{t})$,

where $\hat{\nabla}$ denotes the nabla operator in dimensionless coordinates. The non-dimensional solute concentration $\hat{c}(\hat{\mathbf{x}}, \hat{t})$, dispersion tensor $\hat{\mathbf{n}}$, and conductivity fluctuations $\hat{k}(\hat{\mathbf{y}})$ are defined by

$c(\mathbf{x}, t)=L^{d} \hat{c}\left(\hat{\mathbf{x}} L, \hat{t} \tau_{u}\right), \quad \mathbf{D}=\hat{\mathbf{D}} \bar{u} L, \quad k(\mathbf{y})=\hat{k}(\hat{\mathbf{y}} L)$.

The initial and boundary conditions (6) and (7) are non-dimensionalized accordingly. The non-dimensional correlation length is given by $\hat{l}=l / L$.

Eq. (26) constitutes our working equation in the following. For convenience, we drop the hats, which indicate non-dimensional quantities, in the following.
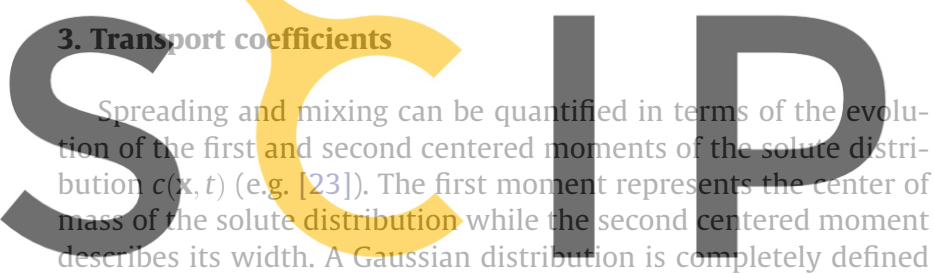
by its first and second moments. Solute distributions in heteroge-

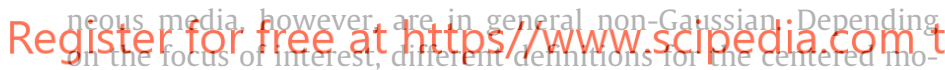
ments and the quantities derived can apply. For risk assessment studies, for example, it may be sufficient to have a rough estimate of the possible extent of a contaminant plume. For reactive transport modeling, in contrast, it is important to have an accurate estimate on the actual spatial distribution of the reactant. Dentz and Carrera [24] suggest and discuss several measures for solute spreading and mixing based on the first and second moments of the transport Green function $g\left(\mathbf{x}, t \mid \mathbf{x}^{\prime}, t^{\prime}\right)$, see (8). In the following we summarize these measures and generalize them for transport in stratified random media within a stochastic modeling approach.

\subsection{Concepts}

Lateral solute transport is completely determined by lateral local dispersion and not affected by spatial heterogeneity. This can be easily seen by projection of transport in the stratified medium onto the plane perpendicular to the direction of stratification by integration of (26) over $x_{1}$, see below. Thus, in the following we focus on transport along the direction of stratification. Local moments are defined in terms of the transport Green function $g\left(\mathbf{x}, t \mid \mathbf{x}^{\prime}, t^{\prime}\right)$ and are given by

$\mu^{(n)}\left(t \mid \mathbf{x}^{\prime}\right)=\int_{\Omega^{d}} \mathrm{~d} \mathbf{x} x_{1}^{n} g\left(\mathbf{x}, t \mid \mathbf{x}^{\prime}, 0\right)$.

The width of the distribution $g\left(\mathbf{x}, t \mid \mathbf{x}^{\prime}, 0\right)$ is given by the local second centered moment

$\kappa^{e}\left(t \mid \mathbf{x}^{\prime}\right)=\mu^{(2)}\left(t \mid \mathbf{x}^{\prime}\right)-\mu^{(1)}\left(t \mid \mathbf{x}^{\prime}\right)^{2}$
The moments of the concentration distribution $c(\mathbf{x}, t)$ given by (26) for the initial condition (6) are defined by

$m^{(n)}(t)=\int_{\Omega^{d}} \mathrm{~d} \mathbf{x} x_{1}^{n} c(\mathbf{x}, t)$.

These "global moments" can be expressed in terms of the local moments as

$m^{(n)}(t)=\int_{\Omega^{d}} \mathrm{~d} \mathbf{x} \rho(\mathbf{x}) \mu^{(n)}(t \mid \mathbf{x})$,

which follows directly from (8). The apparent second centered moment of $c(\mathbf{x}, t)$ is defined by

$\kappa^{a}(t)=m^{(2)}(t)-m^{(1)}(t)^{2}$.

in analogy to (29).

\subsubsection{Single realization}

For a point-like injection, the temporal rate of change of the width of the solute distribution is quantified by the local effective dispersion coefficient (e.g. [24])

$D^{e}\left(t \mid \mathbf{x}^{\prime}\right)=\frac{1}{2} \frac{\mathrm{d}}{\mathrm{d} t} \kappa^{e}\left(t \mid \mathbf{x}^{\prime}\right)$

The local center of mass velocity is given by the time derivative of the first local moment

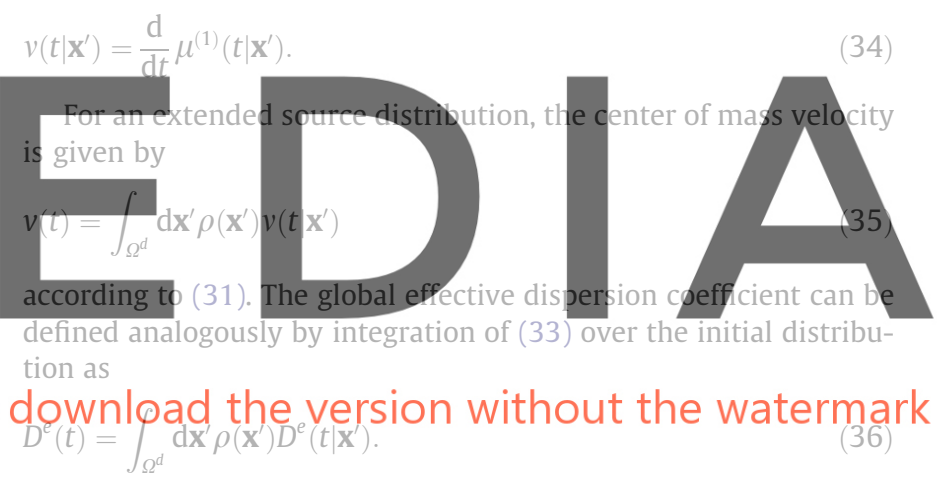

The rate of change of the apparent second centered moment defines the apparent dispersion coefficient

$D^{a}(t)=\frac{1}{2} \frac{\mathrm{d}}{\mathrm{d} t} \kappa^{a}(t)$

As discussed in [24], for pre-asymptotic times, the apparent dispersion coefficient quantifies purely advective spreading of the solute due to velocity contrast within the initial plume. The global effective dispersion coefficient $D^{e}(t)$, in contrast, does not take into account such spreading effects as it is defined as the weighted sum over the local effective dispersion coefficients, which measure solute dispersion originating from the point sources that constitute the initial plume. Thus, advective spreading due to the velocity contrast inside the initial plume can be quantified by the difference between the apparent and the global effective dispersion coefficients

$D^{a}(t)-D^{e}(t)=\frac{1}{2} \frac{\mathrm{d}}{\mathrm{d} t} \int_{\Omega^{d}} \mathrm{~d} \mathbf{x}^{\prime} \rho\left(\mathbf{x}^{\prime}\right)\left[\mu^{(1)}\left(t \mid \mathbf{x}^{\prime}\right)-m^{(1)}(t)\right]^{2}$.

The latter describes the temporal rate of change of the mean squared deviation of the local from the global center of mass positions.

\subsubsection{Ensemble average}

In a stochastic modeling framework, transport coefficients are defined as averages of the single realization coefficients over all realizations of the ensemble of stratified media. As pointed out 
in, e.g. [25], the definition of average dispersion coefficients is not unique and depends on the way the average is taken. This is analogous to the definition of the global effective and apparent dispersion coefficients in the previous section. The two quantities differ in the order, by which the "average" over the initial distribution is taken, see (36) and (37).

In a straightforward manner, we define the average center of mass velocity and local effective dispersion coefficients as the ensemble averages over (34) and (33)

$\bar{v}\left(t \mid \mathbf{X}^{\prime}\right)=\overline{v\left(t \mid \mathbf{X}^{\prime}\right)}, \quad \bar{D}^{e}\left(t \mid \mathbf{X}^{\prime}\right)=\overline{D^{e}\left(t \mid \mathbf{X}^{\prime}\right)}$.

For an extended solute distribution, the average global center of mass velocity and effective dispersion coefficient are given by

$\bar{v}(t)=\int_{\Omega^{d}} \mathrm{~d} \mathbf{x}^{\prime} \rho\left(\mathbf{x}^{\prime}\right) \bar{v}\left(t \mid \mathbf{x}^{\prime}\right), \quad \bar{D}^{e}(t)=\int_{\Omega^{d}} \mathrm{~d} \mathbf{x}^{\prime} \rho\left(\mathbf{x}^{\prime}\right) \bar{D}^{e}\left(t \mid \mathbf{x}^{\prime}\right)$.

The average apparent dispersion coefficient is defined by the ensemble average over (37)

$\bar{D}^{a}(t)=\overline{D^{a}(t)}$

For early times, the apparent dispersion coefficient quantifies spreading due to the velocity contrast within the initial solute distribution, compare (38).

In addition, we will consider the so-called ensemble dispersion coefficient, which describes the temporal rate of change of the longitudinal width of the ensemble averaged Green function $\overline{g\left(\mathbf{x}, t \mid \mathbf{x}^{\prime}, t^{\prime}\right)}$
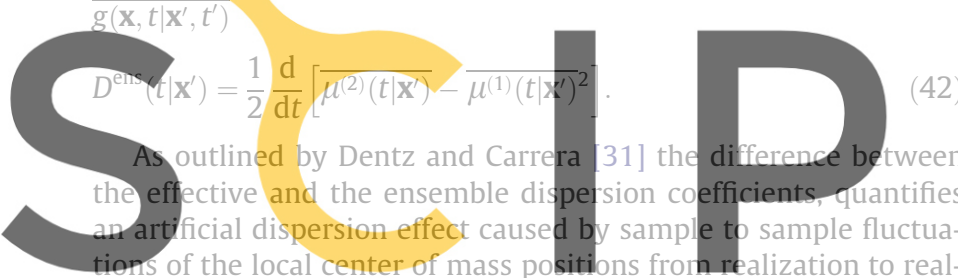

ization of the stratified medium. This unphysical ensemble spreading mechanism is suppressed in the definition of the average

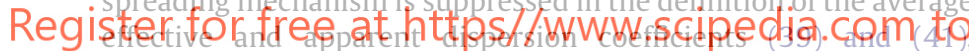
respectively.

The difference between the ensemble and average effective dispersion coefficients, is given by

$D^{\mathrm{ens}}\left(t \mid \mathbf{x}^{\prime}\right)-\bar{D}^{e}\left(t \mid \mathbf{x}^{\prime}\right)=\frac{1}{2} \frac{\mathrm{d}}{\mathrm{d} t}\left[\overline{\mu^{(1)}\left(t \mid \mathbf{x}^{\prime}\right)^{2}}-\overline{\mu^{(1)}\left(t \mid \mathbf{x}^{\prime}\right)^{2}}\right]$,

i.e., the two coefficients differ in the fluctuations of the center of mass from realization to realization.

For an extended solute distribution, the ensemble dispersion coefficient is given by

$D^{\text {ens }}(t)=\int_{\Omega^{d}} \mathrm{~d} \mathbf{x}^{\prime} \rho\left(\mathbf{x}^{\prime}\right) D^{\text {ens }}\left(t \mid \mathbf{x}^{\prime}\right)$.

The difference between the ensemble and average apparent dispersion coefficients is given by

$D^{\mathrm{ens}}(t)-\bar{D}^{a}(t)=\frac{1}{2} \frac{\mathrm{d}}{\mathrm{d} t} \int_{\Omega^{d}} \mathrm{~d} \mathbf{x}^{\prime} \rho\left(\mathbf{x}^{\prime}\right) \overline{\mu^{(1)}\left(t \mid \mathbf{x}^{\prime}\right)\left[m^{(1)}(t)-\overline{\mu^{(1)}\left(t \mid \mathbf{x}^{\prime}\right)}\right]}$.

The dispersion concepts for single realizations and the ensemble of realizations of the stratified medium are summarized in Table 1.

\subsection{Transport coefficients: single realization}

Having found expressions for the local moments using axial moment equations, see Appendix A, we can write down the expressions for the local center of mass velocity and effective dispersion coefficients. They are given by
Table 1

Dispersion concepts

\begin{tabular}{lllll}
\hline Single realization & Eq. & Ensemble average & Eq. & Concept \\
\hline$D^{a}(t)$ & $(37)$ & $\bar{D}^{a}(t)$ & $(41)$ & Apparent dispersion \\
$D^{e}\left(t \mid \mathbf{x}^{\prime}\right)$ & $(33)$ & $\bar{D}^{e}\left(t \mid \mathbf{x}^{\prime}\right)$ & $(39)$ & Local effective dispersion \\
$D^{e}(t)$ & $(36)$ & $\bar{D}^{e}(t)$ & $(40)$ & Global effective dispersion \\
$D_{L}, D_{\mathrm{T}}$ & $(5)$ & $\mathrm{N} / \mathrm{A}$ & - & Local dispersion \\
$D^{*}$ & $(1)$ & $\bar{D}^{*}$ & $(56)$ & Taylor dispersion \\
N/A & - & $D^{\text {ens }}\left(t \mid \mathbf{x}^{\prime}\right)$ & $(42)$ & Local ensemble dispersion \\
N/A & - & $D^{\text {ens }}(t)$ & $(44)$ & Global ensemble dispersion \\
\hline
\end{tabular}

$v\left(t \mid \mathbf{x}^{\prime}\right)=1-\int_{\Omega} \mathrm{d} \mathbf{y}^{\prime \prime} k\left(\mathbf{y}^{\prime \prime}\right) c_{0}\left(\mathbf{y}^{\prime \prime}, t \mid \mathbf{y}^{\prime}\right)$,

$$
\begin{aligned}
D^{e}\left(t \mid \mathbf{x}^{\prime}\right)= & D_{L}+\int_{\Omega} \mathrm{d} \mathbf{y}^{\prime \prime} \int_{\Omega} \mathrm{d} \mathbf{y} \int_{0}^{t} \mathrm{~d} t^{\prime} k\left(\mathbf{y}^{\prime \prime}\right) k(\mathbf{y}) \\
& \times\left[c_{0}\left(\mathbf{y}^{\prime \prime}, t-t^{\prime} \mid \mathbf{y}\right)-c_{0}\left(\mathbf{y}^{\prime \prime}, t \mid \mathbf{y}^{\prime}\right)\right] c_{0}\left(\mathbf{y}, t^{\prime} \mid \mathbf{y}^{\prime}\right) .
\end{aligned}
$$

The global center of mass velocity and effective dispersion coefficient are obtained by integration of (46) and (47) over the initial distribution. The apparent dispersion coefficient is given by

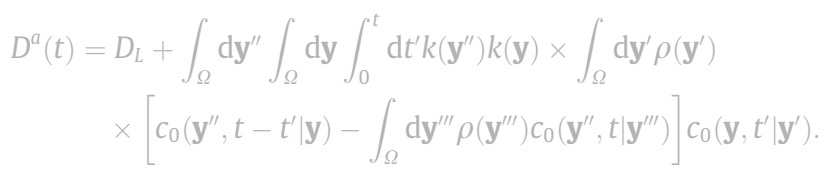

$(48)$

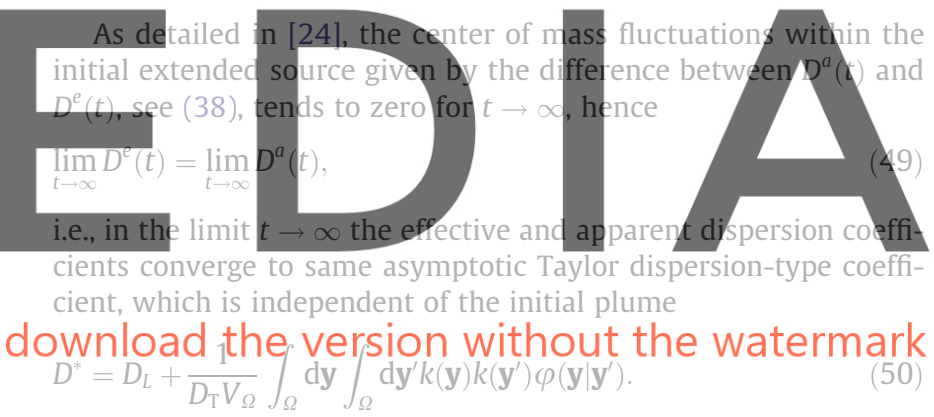

The $\varphi\left(\overline{\mathbf{y}} \mid \overline{\mathbf{y}}^{\prime}\right)$ solves the steady state diffusion equation

$\frac{1}{V_{\Omega}}-\nabla_{\mathbf{y}}^{2} \varphi\left(\mathbf{y} \mid \mathbf{y}^{\prime}\right)=\delta\left(\mathbf{y}-\mathbf{y}^{\prime}\right)$,

for Neumann boundary conditions.

\subsection{Transport coefficients: ensemble}

By performing the ensemble average over (46), we straightforwardly obtain for the center of mass velocity

$\bar{v}\left(t \mid \mathbf{x}^{\prime}\right)=1$,

i.e., there is no disorder-induced contribution to the ensemble averaged center of mass velocity. This is different for the average dispersion coefficients.

Inserting (A.11) and (A.12) for the local moments in (42) and (39) for the ensemble and effective dispersion coefficients, we obtain

$$
\begin{aligned}
& D^{\mathrm{ens}}\left(t \mid \mathbf{y}^{\prime}\right)= D_{L}+\sigma^{2} \int_{\Omega} \mathrm{d} \mathbf{y}^{\prime \prime} \int_{\Omega} \mathrm{d} \mathbf{y} \int_{0}^{t} \mathrm{~d} t^{\prime} C\left(\mathbf{y}-\mathbf{y}^{\prime \prime}\right) \\
& \times c_{0}\left(\mathbf{y}^{\prime \prime}, t-t^{\prime} \mid \mathbf{y}\right) c_{0}\left(\mathbf{y}, t^{\prime} \mid \mathbf{y}^{\prime}\right), \\
& \bar{D}^{e}\left(t \mid \mathbf{y}^{\prime}\right)= D^{\mathrm{ens}}\left(t \mid \mathbf{y}^{\prime}\right)-\sigma^{2} \int_{\Omega} \mathrm{d} \mathbf{y} \int_{\Omega} \mathrm{d} \mathbf{y}^{\prime \prime} C\left(\mathbf{y}-\mathbf{y}^{\prime \prime}\right) \times c_{0}\left(\mathbf{y}^{\prime \prime}, t \mid \mathbf{y}^{\prime}\right) \\
& \int_{0}^{t} \mathrm{~d} t^{\prime} c_{0}\left(\mathbf{y}, t^{\prime} \mid \mathbf{y}^{\prime}\right) .
\end{aligned}
$$


The corresponding expressions for extended initial plumes are obtained by integration over the source distribution according to (40) and (44).

For the average apparent dispersion coefficient (41), we obtain

$$
\begin{aligned}
\bar{D}^{a}(t)= & D^{\mathrm{ens}}(t)-\sigma^{2} \int_{\Omega} \mathrm{d} \mathbf{y}^{\prime \prime} \int_{\Omega} \mathrm{d} \mathbf{y} C\left(\mathbf{y}-\mathbf{y}^{\prime \prime}\right) \\
& \int_{\Omega} \mathrm{d} \mathbf{y}^{\prime \prime \prime} \rho\left(\mathbf{y}^{\prime \prime \prime}\right) c_{0}\left(\mathbf{y}^{\prime \prime}, t \mid \mathbf{y}^{\prime \prime \prime}\right) \int_{0}^{t} \mathrm{~d} t^{\prime} \int_{\Omega} \mathrm{d} \mathbf{y}^{\prime} \rho\left(\mathbf{y}^{\prime}\right) c_{0}\left(\mathbf{y}, t^{\prime} \mid \mathbf{y}^{\prime}\right) .
\end{aligned}
$$

The average asymptotic Taylor dispersion-type coefficient is given by

$\bar{D}^{*}=D_{L}+\frac{\sigma^{2}}{D_{\mathrm{T}} V_{\Omega}} \int_{\Omega} \mathrm{d} \mathbf{y} \int_{\Omega} \mathrm{d} \mathbf{y}^{\prime} C\left(\mathbf{y}-\mathbf{y}^{\prime}\right) \varphi\left(\mathbf{y} \mid \mathbf{y}^{\prime}\right)$.

\subsection{Explicit analytical solutions for $d=2$-dimensions}

Having defined the $d=2$-dimensional model medium in Section 2, and in particular the conductivity correlation function (16), we can explicitly evaluate the expressions for the average dispersion coefficients. We consider two initial conditions; point-like injection at the position $\left(0, y^{\prime}\right)^{\mathrm{T}}$, i.e.

$\rho(\mathbf{x})=\delta(x) \delta\left(y-y^{\prime}\right)$

and a line source that extends over the whole medium cross-section at $x=0$

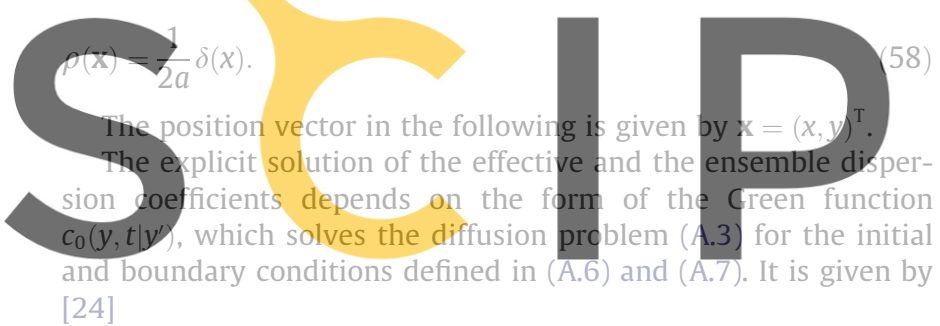

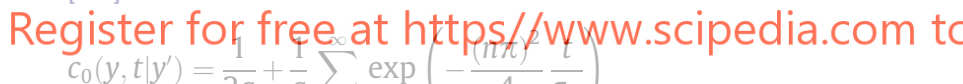

$$
\begin{aligned}
c_{0}\left(y, t \mid y^{\prime}\right)= & \frac{1}{2 a}+\frac{1}{a} \sum_{n=1} \exp \left(-\frac{1 \pi}{4} \frac{}{\tau_{D}}\right) \\
& \times \cos \left[\frac{n \pi(y+a)}{2 a}\right] \cos \left[\frac{n \pi\left(y^{\prime}+a\right)}{2 a}\right] .
\end{aligned}
$$

The dispersion time scale $\tau_{\mathrm{D}}$, given by (2), measures the time for transport by local dispersion over the medium cross-section.

Inserting the spatial correlation function (16) and Green function (59) into the general expressions (53) and (54), we obtain for the ensemble and average effective dispersion coefficients

$$
\begin{aligned}
D^{\mathrm{ens}}\left(t \mid y^{\prime}\right)= & D_{L}+\frac{\sigma^{2} l}{2 a} t+\frac{2 \sigma^{2} l}{a} \tau_{\mathrm{D}} \sum_{n=1}^{\infty} \frac{1}{(n \pi)^{2}} \\
& \times\left[1-\exp \left(-\frac{(n \pi)^{2}}{4} \frac{t}{\tau_{\mathrm{D}}}\right)\right]+\frac{2}{3 a} \sigma^{2} l \tau_{\mathrm{D}} \sum_{m} \frac{(-1)^{\mathrm{m}}}{(m \pi)^{2}} \\
& \times\left[\exp \left(-\frac{(m \pi)^{2}}{4} \frac{t}{\tau_{\mathrm{D}}}\right)-\exp \left(-(m \pi)^{2} \frac{t}{\tau_{\mathrm{D}}}\right)\right] \\
& \times \cos \left(\frac{m \pi y^{\prime}}{a}\right),
\end{aligned}
$$

$$
\begin{aligned}
\bar{D}^{e}\left(t \mid y^{\prime}\right)= & D^{\text {ens }}\left(t \mid y^{\prime}\right)-\frac{\sigma^{2} l}{2 a} t-\frac{2 \sigma^{2} l}{a} \tau_{\mathrm{D}} \sum_{n=1}^{\infty} \frac{1}{(n \pi)^{2}} \\
& \times\left[\exp \left(-\frac{(n \pi)^{2}}{4} \frac{t}{\tau_{\mathrm{D}}}\right)-\exp \left(-\frac{(n \pi)^{2}}{2} \frac{t}{\tau_{\mathrm{D}}}\right)\right] \\
& \times\left[1+(-1)^{n} \cos \left(\frac{n \pi y^{\prime}}{a}\right)\right] .
\end{aligned}
$$

Explicit expressions for the instantaneous line source are obtained by integration of (60) and (61) over the vertical cross-section of the two-dimensional medium. This yields

$$
\begin{aligned}
D^{\mathrm{ens}}(t)= & D_{L}+\frac{\sigma^{2} l}{2 a} t+\frac{2 \sigma^{2} l}{a} \tau_{\mathrm{D}} \\
& \sum_{n=1}^{\infty} \frac{1}{(n \pi)^{2}}\left[1-\exp \left(-\frac{(n \pi)^{2}}{4} \frac{t}{\tau_{\mathrm{D}}}\right)\right], \\
\bar{D}^{e}(t)= & D_{L}+\frac{2 \sigma^{2} l}{a} \tau_{\mathrm{D}} \sum_{n=1}^{\infty} \frac{1}{(n \pi)^{2}}\left[1-\exp \left(-\frac{(n \pi)^{2}}{4} \frac{t}{\tau_{\mathrm{D}}}\right)\right]^{2}
\end{aligned}
$$

for the ensemble and average global effective dispersion coefficients, respectively. For the average apparent dispersion coefficients we obtain by inserting (16) into (55)

$\bar{D}^{a}(t)=D_{L}+\frac{2 \sigma^{2} l}{a} \tau_{\mathrm{D}} \sum_{n=1}^{\infty} \frac{1}{(n \pi)^{2}}\left[1-\exp \left(-\frac{(n \pi)^{2}}{4} \frac{t}{\tau_{\mathrm{D}}}\right)\right]$

The average Taylor dispersion-type coefficient (56) can be obtained as the asymptotic long time value of the average effective dispersion coefficient

$\bar{D}^{*}=D_{L}+\frac{\sigma^{2} l \tau_{\mathrm{D}}}{3 a}$.

The transverse dimension clearly determines the asymptotic long time behavior of the dispersion coefficients. In the limit of

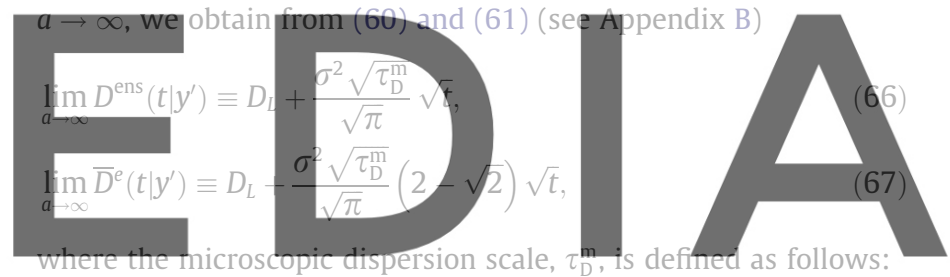

\section{dơnhload the version without the waterifiark}

and quantifies the time for the vertical solute spreading over a stratum.

Note that neither the average local effective nor the ensemble dispersion coefficients depend on the source location anymore. As a consequence, the global effective dispersion coefficient does not depend on the source and is identical to the local effective dispersion coefficient. Furthermore, in this limit the average apparent dispersion coefficient (64) for the line source (now infinitely extended) is identical to the ensemble dispersion coefficient

$\lim _{a \rightarrow \infty} D^{\text {ens }}(t) \equiv \lim _{a \rightarrow \infty} \bar{D}^{a}(t)$.

Note that in contrast to the behavior observed in a confined medium where in the limit of $t \rightarrow \infty$, or strictly speaking when the solute has sampled the hole medium by local transverse dispersion for times $t \gg \tau_{\mathrm{D}}, \bar{D}^{a}(t)$ and $\bar{D}^{e}(t)$ converge to the same asymptotic Taylor dispersion coefficient, (49), here for a infinitely extended initial plume the apparent and the global effective dispersion coefficients do not converge at large times because $\tau_{\mathrm{D}} \rightarrow \infty$. This means first that the limits $a \rightarrow \infty$, and $t \rightarrow \infty$, are not commutative. Furthermore, as $\tau_{\mathrm{D}} \rightarrow \infty$ the complete transverse mixing is never reached for a infinite medium, which in turns leads to the non-Fickian behavior of the effective dispersion coefficients.

\subsection{Numerical random walk simulations}

The numerical solution of the transport problem using random walk simulations are based on the Langevin equation which is 
associated with the transport equation (26), see, e.g. [32]. In discrete time, the equation of a solute particle starting at $\mathbf{x}(0)=\left(0, \mathbf{y}^{\prime}\right)$ is given by

$x^{(i, r)}\left(t+\Delta t \mid \mathbf{y}^{\prime}\right)=x^{(i, r)}(t)+\left[1-k^{(r)}\left(\mathbf{y}^{(i, r)}\left(t \mid \mathbf{y}^{\prime}\right)\right)\right] \Delta t+\sqrt{2 D_{L} \Delta t} \xi_{N}^{(i)}$,

$\mathbf{y}^{(i, r)}\left(t+\Delta t \mid \mathbf{y}^{\prime}\right)=\mathbf{y}^{(i, r)}\left(t \mid \mathbf{y}^{\prime}\right)+\sqrt{2 D_{\mathrm{T}} \Delta t} \boldsymbol{\eta}_{N}^{(i)}$

where $\xi_{N}^{(i)}$ and $\boldsymbol{\eta}_{N}^{(i)}$ are mutually independent Gaussian random vectors with zero mean and unit variance; $k^{(r)}(y)$ is the $r$ th realization of $k(\mathbf{y}) ; x^{(i, r)}\left(t \mid \mathbf{y}^{\prime}\right)$ and $\mathbf{y}^{(i, r)}\left(t \mid \mathbf{y}^{\prime}\right)$ denote the particle trajectory starting from $\mathbf{x}(0)$ in the $i$ th noise and $r$ th disorder realization. The impermeable horizontal walls are modeled as reflecting boundaries. The random fluctuations of the conductivity field are modeled by (14).

The local moments $\mu^{(n, r)}\left(t \mid \mathbf{y}^{\prime}\right)$ in the $r$ th disorder realization are derived from averages of the particle trajectories over all noise realizations as (e.g., [241)

\section{$\mu^{(n, r)}\left(t \mid \mathbf{y}^{\prime}\right)=\frac{1}{N} \sum_{i=1}^{N}\left[x^{(i, r)}\left(t \mid \mathbf{y}^{\prime}\right)\right]$}

where $N$ is the total number of noise realizations. The initial positions $\mathbf{y}^{(j)}$, which are distributed uniformly over the medium cross-section according to $\mathbf{y}^{(j)}=j \Delta \mathbf{y}^{\prime}$, where $j=-M, \ldots, M$ with $M \Delta y^{\prime}=a$. The global moments in the $r$-th disorder realization then are obtained by summation of (72) over all initial positions as

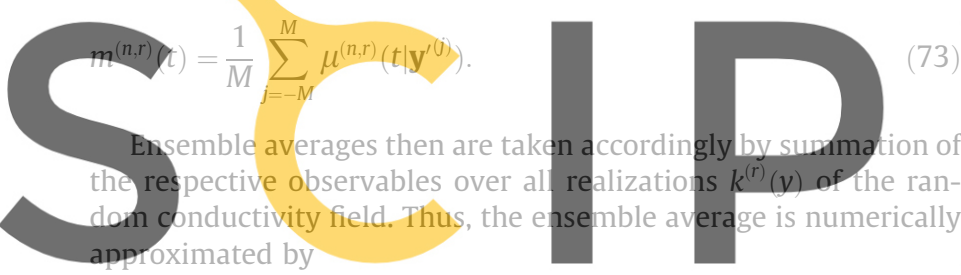

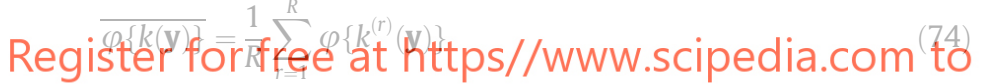

with $\varphi\{k(\mathbf{y})\}$ an arbitrary functional of $k(y)$ and $R$ the total number of disorder realizations.

The $d=2$-dimensional medium under consideration, see Section 2, consists of 100 strata of equal thickness $l=1$, the halfwidth of the medium is given by $a=50$. The time discretization for the random walk simulations is $\Delta t=10^{-1}$, the transverse and longitudinal local dispersion coefficients are given by $D_{\mathrm{T}}=D_{L}=1$. The line source consists of $M=200$ points that are uniformly distrib- uted over the medium cross-section, the number of injected particles at each point is 50 . The ensemble average is taken over $R=10^{3}$ realizations of the random conductivity field.

\section{Effective mixing and spreading}

Here, we study the effective mixing and spreading in terms of the observables defined in the previous section using the numerical random walk simulations and explicit analytical expressions for the transport coefficients.

\subsection{Point-like initial distribution}

We investigate the temporal behavior of the dispersion coefficients $\bar{D}^{e}\left(t \mid y^{\prime}\right)$ and $D^{\text {ens }}\left(t \mid y^{\prime}\right)$, for different dispersion time scales $\tau_{\mathrm{D}}$, and for different vertical initial positions $y^{\prime}$.

Fig. 2 illustrates a particle distribution evolving from an instantaneous injection at $y^{\prime}=0$ in one given realization of the stratified medium. The snap shots of the spatial distribution are taken after $t=10^{-3} \tau_{\mathrm{D}}, t=10^{-2} \tau_{\mathrm{D}}, t=10^{-1} \tau_{\mathrm{D}}$, and $t=\tau_{\mathrm{D}}$

We distinguish two relevant dispersion time scales; the dispersion scale $\tau_{\mathrm{D}}$, (2), which quantifies solute mixing over a vertical medium cross-section, and the microscopic dispersion scale $\tau_{\mathrm{D}}^{\mathrm{m}}$, (68), which quantifies the time for the vertical solute mixing over a stratum. Thus, $\tau_{\mathrm{D}}^{\mathrm{m}}$ sets the relevant scale for the activation of the transverse conductivity contrast as a macroscopic longitudinal

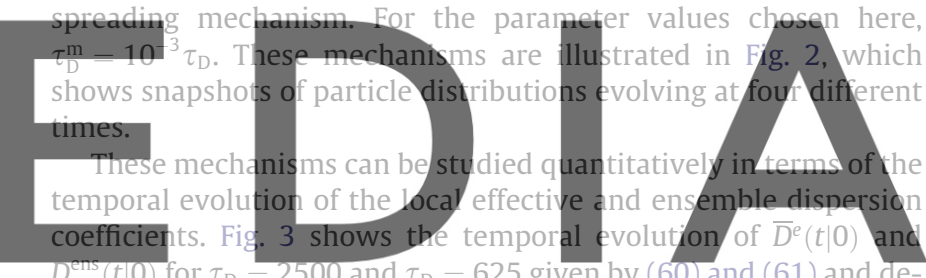
rived from numerical random walk simulations. For times smaller

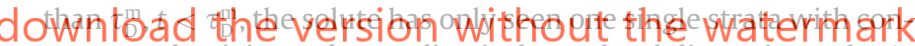
stant conductivity and spreading is due to local dispersion only. At times of the order of the microscopic dispersion time scale $\tau_{\mathrm{D}}^{\mathrm{m}}$ the solute starts sampling the vertical conductivity contrast. In the time regime $\tau_{\mathrm{D}}^{\mathrm{m}} \ll t \ll \tau_{\mathrm{D}}$, the dispersion coefficients display the characteristic $\sqrt{t}$ behavior [1] because the medium looks infinite for the solute. In the asymptotic long time limit of times $t \gg \tau_{\mathrm{D}}$, the effective dispersion coefficient converges to its constant long time value given by the constant Taylor dispersion coefficient (65). The ensemble dispersion coefficient $D^{\text {ens }}(t \mid 0)$ is consistently
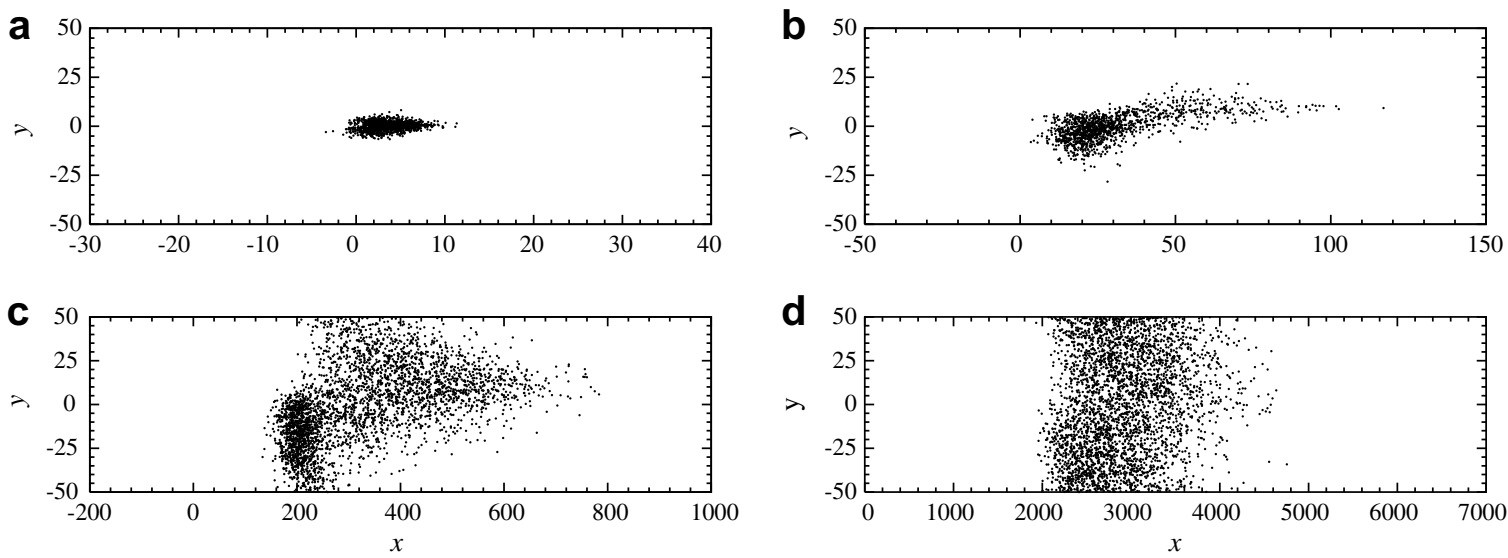

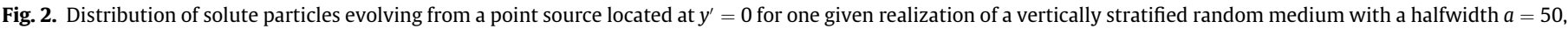
strata thickness $l=1$, and $D_{L}=D_{\mathrm{T}}=1$, after (a) $t=10^{-3} \tau_{\mathrm{D}}$, (b) $t=10^{-2} \tau_{\mathrm{D}}$, (c) $t=10^{-1} \tau_{\mathrm{D}}$, and (d) $t=\tau_{\mathrm{D}}$. 


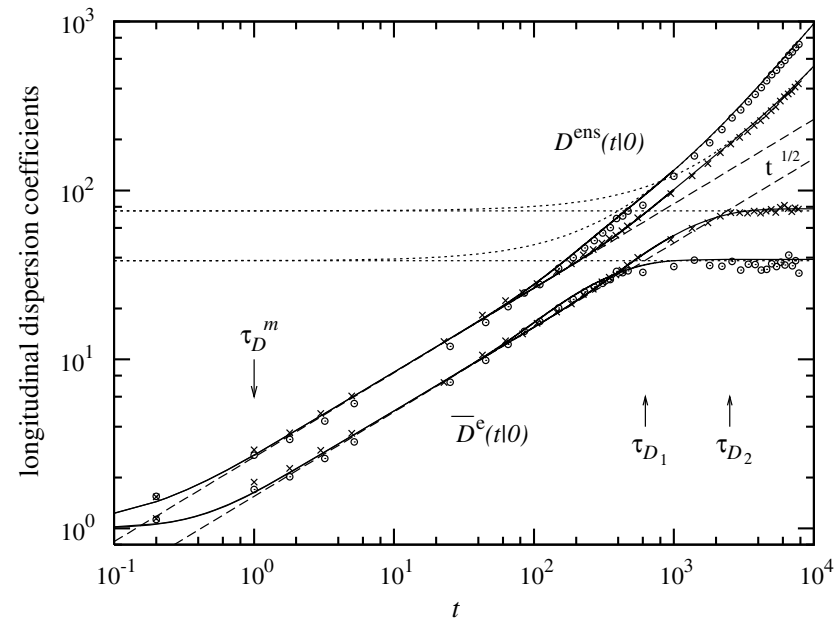

Fig. 3. Temporal behavior of the local effective and ensemble dispersion coefficients evolving from a point source at $y^{\prime}=0$, for two different dispersion time scales, $\tau_{D_{1}}=625$, and $\tau_{D_{2}}=2500$ in a vertically stratified random media with $a=25, a=50$, respectively, $l=1$, and $D_{L}=D_{\mathrm{T}}=1$. The solid lines describe the $D^{\text {ens }}\left(t \mid y^{\prime}\right)$ and $\bar{D}^{e}\left(t \mid y^{\prime}\right)$ behavior given by (60) and (61), respectively. For the intermediate time regime $\tau_{\mathrm{D}}^{\mathrm{m}} \ll t \ll \tau_{\mathrm{D}}$, the $\sqrt{t}$ behavior given by (66) and (67) is showed in dashed lines. The doted lines describe the long time behavior, given by (75) and (65). Numerical random walk simulation for $\tau_{D_{1}}$ in unfilled dots, for $\tau_{D_{2}}$ in crossed lines.

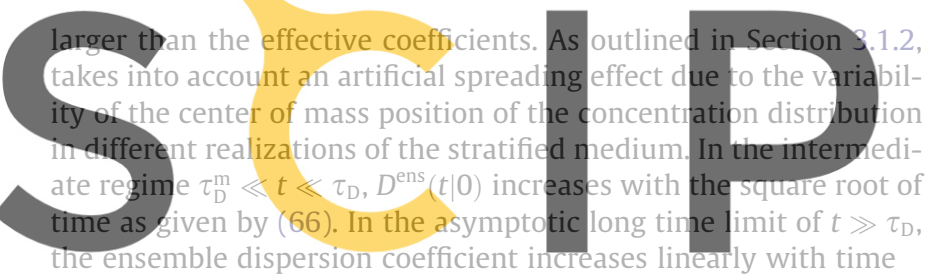

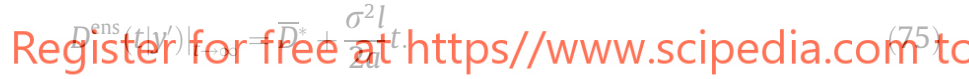

This linear increase is due to persistent center of mass fluctuations, which increase quadratically with time. This can be seen as follows: the center of mass position in a given realization is given by (A.11). Thus, the center of mass fluctuation is given by

$$
\begin{aligned}
\delta \mu^{(1)}\left(t \mid \mathbf{y}^{\prime}\right) & =\overline{\mu^{(1)}\left(t \mid \mathbf{y}^{\prime}\right)}-\mu^{(1)}\left(t \mid \mathbf{y}^{\prime}\right) \\
& =\int_{-a}^{a} \mathrm{~d} \mathbf{y}^{\prime \prime} \int_{0}^{t} \mathrm{~d} t^{\prime} k\left(\mathbf{y}^{\prime \prime}\right) c_{0}\left(y^{\prime \prime}, t^{\prime} \mid \mathbf{y}^{\prime}\right) .
\end{aligned}
$$

In the limit $t \gg \tau_{\mathrm{D}}$, the Green function $c_{0}\left(\mathbf{y}^{\prime \prime}, t^{\prime} \mid \mathbf{y}^{\prime}\right)$ tends to $1 /(2 a)$, as in this limit the solute is uniformly distributed over the medium cross-section. Thus, $\delta \mu^{(1)}\left(t \mid \mathbf{y}^{\prime}\right)$ is given by

$\delta \mu^{(1)}\left(t \mid \mathbf{y}^{\prime}\right)=\left\langle k\left(\mathbf{y}^{\prime}\right)\right\rangle t$,

where the angular brackets denote the spatial average over the medium cross-section, see Section 2.2. Thus, the variance of the center of mass position is given in terms of the variance (18) of the spatial average conductivity as

$\overline{\delta \mu^{(1)}\left(t \mid \mathbf{y}^{\prime}\right)^{2}}=\frac{\sigma^{2} l}{2 a} t^{2}$.

The difference between the effective and ensemble coefficients, see (43), is given by half the temporal rate of change of the latter and explains the linear increase of $D^{\text {ens }}\left(t \mid y^{\prime}\right)$ for $t \gg \tau_{\mathrm{D}}$.

Note that the center of mass fluctuations decrease with increasing vertical extent, or vertical sampling volume. This mechanism is illustrated in Fig. 4. The difference between the global ensemble and the apparent dispersion coefficients obtained by numerical random walk simulations for three different vertically extended

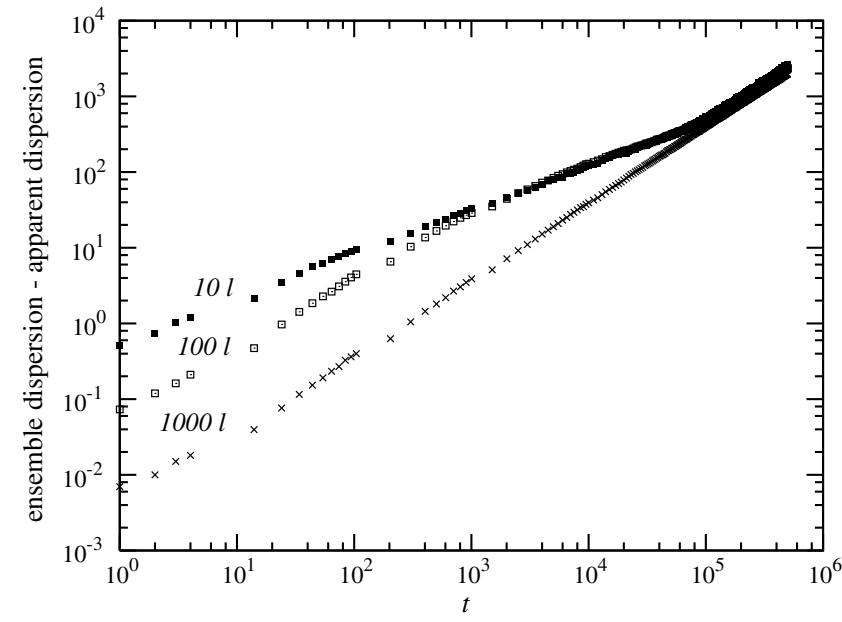

Fig. 4. Numerical results of the differences between the global ensemble and apparent dispersion coefficients for three extended sources of $10 l, 100 l$, and a line source that extends over the whole medium cross-section of $1000 \mathrm{l}$

source distributions of $10 l, 10^{2} l$ and a line source that extends over the whole medium cross-section of $10^{3} \mathrm{l}$ are shown. For an extended source distribution, the difference between the global ensemble and apparent dispersion coefficients quantifies the fluc-

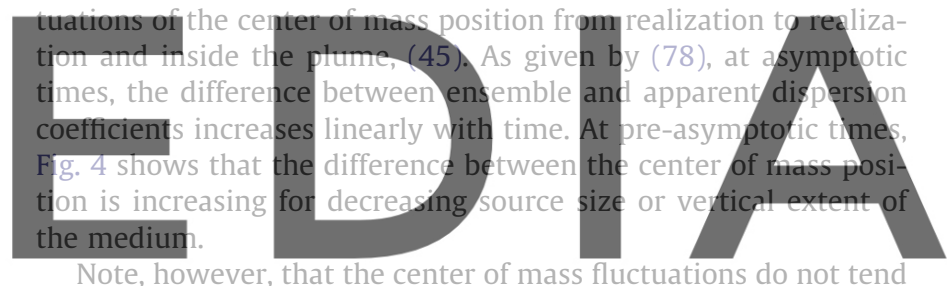

zero in the limit of the infinite medium. Eq. (78) is based on the

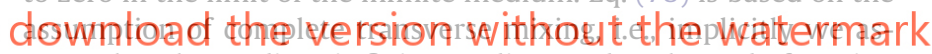
sume that the medium is finite. As discussed at the end of Section 3.4 the limits $a \rightarrow \infty$ and $t \rightarrow \infty$ do not commute. Thus, the fact that expression (78) tends to zero for $a \rightarrow \infty$ does not mean that the center of mass fluctuations die out in this limit because $\tau_{\mathrm{D}} \rightarrow \infty$, and the transverse mixing condition can never be fulfilled. The center of mass fluctuations in the limit of the infinite medium are manifested in the difference between $\bar{D}^{\text {ens }}(t)$ and $\bar{D}^{e}(t)$ for the infinite medium given in (66) and (67), respectively, for which

$\lim _{a \rightarrow \infty} \overline{\delta \mu^{(1)}(t)^{2}}=\frac{2}{3} \frac{\sigma^{2} \sqrt{\tau_{\mathrm{D}}^{\mathrm{m}}}}{\sqrt{\pi}}(1-\sqrt{2}) t^{3 / 2}$.

The center of mass fluctuations are not vanishing, but their growth rate is smaller; for the confined medium, the growth rate increases linearly, for the infinite medium it increases only with the square root of time.

In the following, we study the effect of the position of the solute injection point on effective transport. Fig. 5 illustrates the temporal behavior of a distribution of solute particles evolving from a point source located at $y^{\prime}=0.9 a$ for a given realization of the stratified medium. The medium analyzed here, is the same as in Fig. 2. For point sources starting in the vicinity of the horizontal boundaries, at early times, $t \geqslant \tau_{\mathrm{D}}^{\mathrm{m}}$, the solute particles experience the layer to layer permeability variations by transverse local dispersion in a non-symmetrical way due to reflection at the medium boundaries. Hence, the time needed for a solute particle to sample the transverse variability of the velocity field is larger than when starting at $y^{\prime}=0$. This difference for the distribution starting at different vertical locations is clearly shown in Figs. $2 c$ and $5 c$, for $t=10^{-1} \tau_{\mathrm{D}}$. The particle distribution for $y^{\prime}=0$, is more uniform 

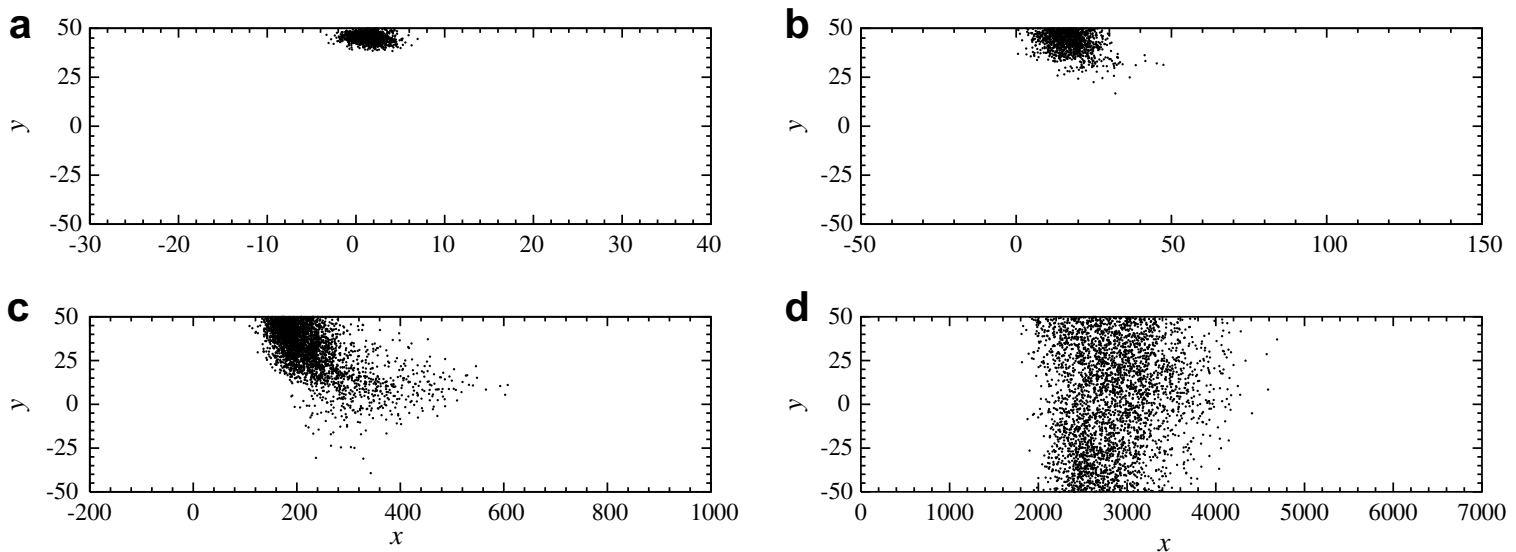

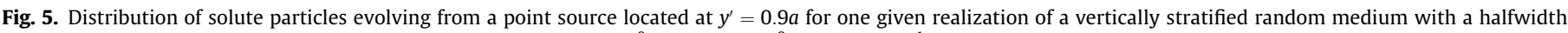
$a=50$, equal strata thickness $l=1$, and $D_{L}=D_{\mathrm{T}}=1$, after (a) $t=10^{-3} \tau_{\mathrm{D}}$, (b) $t=10^{-2} \tau_{\mathrm{D}}$, (c) $t=10^{-1} \tau_{\mathrm{D}}$, and (d) $t=\tau_{\mathrm{D}}$

than for $y^{\prime}=0.9 a$, and it occupies almost the entire medium crosssection. For times of the order of or larger than the dispersion time scale $t \geqslant \tau_{\mathrm{D}}$, the solute particles are distributed uniformly over the medium cross-section in both cases; the memory of the initial distribution is wiped out.

This behavior is again reflected in the temporal evolution of the

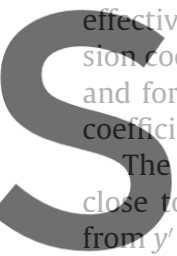
ve dispersion coefficients show efficient $\bar{D}^{e}\left(t \mid y^{\prime}\right)$ evolves slov and for times large compared to coeficients converge to the same slower sampling of the cor the boundaries compared to $=0$ is also reflected by the en
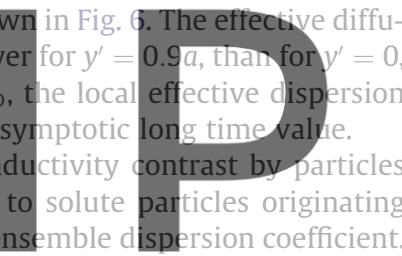

Due to the slower sampling for $y^{\prime}=0.9$, there is a bigger contrast between the mean flow velocity in each realization and the ensem-

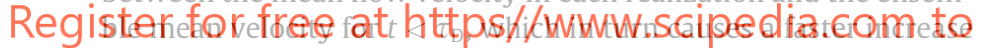
of $D^{\text {ens }}\left(t \mid y^{\prime}\right)$, see Fig. 6. For $t>\tau_{\mathrm{D}}$ both ensemble dispersion coefficients evolve linearly to (75), independently of the source location.

\subsection{Vertical line source}

Here, we investigate mixing and spreading for a solute that evolves from the instantaneous uniform line source (58). For one given realization of the stratified medium, solute spreading and mixing are quantified by the effective and apparent dispersion coefficients (33) and (37), respectively. The time behavior of $D^{e}(t)$ and $D^{a}(t)$ is evaluated numerically using random walk simulations. Fig. 7 shows $D^{e}(t)$ and $D^{a}(t)$ normalized by their respective asymptotic long time values. The apparent and effective coefficients evolve in a qualitatively and quantitatively different way. As outlined in Section 3.1.1, the apparent dispersion coefficient quantifies the center of mass fluctuations within the extended source, which are suppressed by the definition of the effective dispersion coefficient, see (38). Hence, for $t<\tau_{\mathrm{D}}$, the conductivity contrast along the extended source leads to a faster increase of $D^{a}(t)$. The effective dispersion coefficient measures the average mixing along the source distribution caused by the interaction of transverse local dispersion and the vertical conductivity contrast. At times $t \geqslant \tau_{\mathrm{D}}$ both $D^{a}(t)$ and $D^{e}(t)$ evolve towards their common asymptotic long time value given by $D^{*},(50)$.

The behavior of the average coefficients $\bar{D}^{e}(t)$ and $\bar{D}^{a}(t)$, given by (63) and (64), respectively, reflects well the behavior observed in a single realization. Fig. 7 shows $\bar{D}^{e}(t)$ and $\bar{D}^{a}(t)$ normalized by their asymptotic long time value compared to their single realization counterparts for vertical line source of $10^{2} l$.

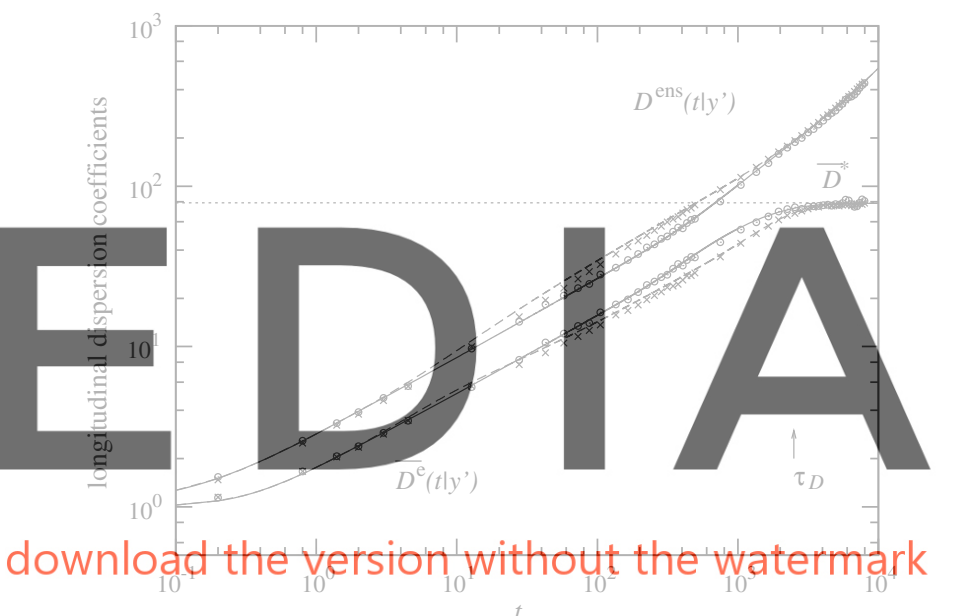

Fig. 6. Time behavior of the local effective and ensemble dispersion coefficients evolving from two point sources located at $y^{\prime}=0$, in solid lines, and $y^{\prime}=0.9 a$, in dashed lines, in a vertically stratified random media with $a=50, l=1$, and $D_{L}=D_{\mathrm{T}}=1$. The dotted line describes the average asymptotic long time value $\bar{D}^{*}$ given by (65). The results of the corresponding numerical random walk simulations are denoted by crosses $\left(y^{\prime}=0.9 a\right)$ and circles $\left(y^{\prime}=0\right)$.

Due to the finiteness of the medium, the asymptotic Taylor dispersion-type coefficient $D^{*},(50)$, varies from realization to realization of the stratified medium. The quantification of uncertainty due to sample to sample fluctuations as well as the self-averaging behavior of the effective dispersion coefficient is work in progress.

\subsection{Extended source distributions}

In the previous sections, we focused on effective mixing and spreading for point-like injections and for solute sources distributed over the full medium cross-section. Here, we study the influence of source size smaller than the medium cross-section on effective mixing and spreading in a confined stratified medium using systematic numerical random walk simulations.

The $d=2$-dimensional model under consideration here consists of $10^{3}$ strata of equal thickness $l=1$. The time discretization is $\Delta t=1$, the transverse and longitudinal local dispersion are given by $D_{\mathrm{T}}=D_{L}=1$. We investigate in the following sources of vertical extensions of $10 l, 10^{2} l$ and a line source that extends over the whole medium cross-section of $10^{3} l$. The total number of injected 


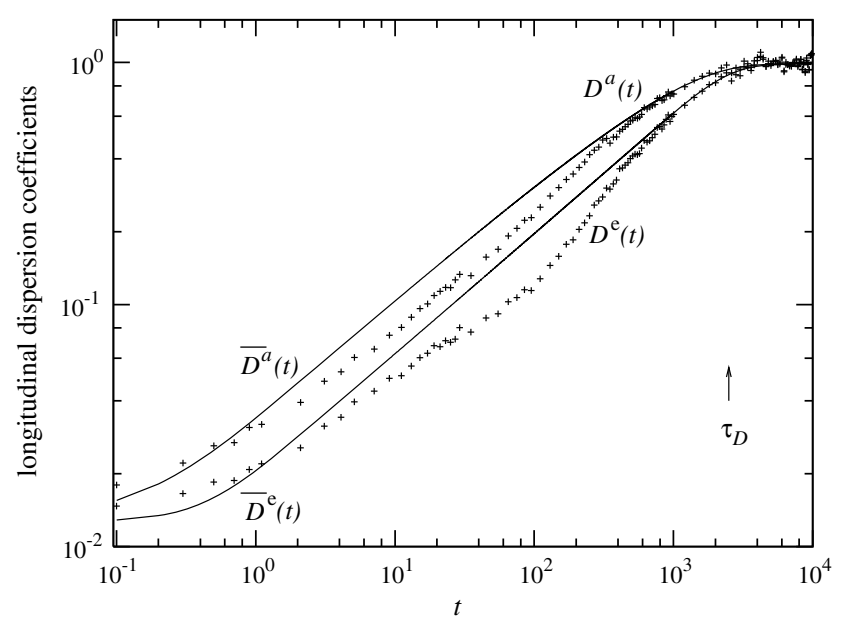

Fig. 7. Comparison between the normalized average effective $\bar{D}^{e}(t) / \bar{D}^{*}$, and apparent $\bar{D}^{a}(t) / \bar{D}^{*}$, dispersion coefficients, with their single realization normalized counterparts, $D^{e}(t) / D^{*}$ and $D^{a}(t) / D^{*}$, respectively, for a vertical line source of $10^{2}$ The temporal behavior of $\bar{D}^{e}(t)$, and $\bar{D}^{a}(t)$ given by (63) and (64), is denoted by solid lines. Numerical random walk simulations of $D^{e}(t)$ and $D^{a}(t)$ are denoted by dots.

particles in each case was $10^{4}$. The ensemble average was taken over $R=10^{2}$ realizations of the random conductivity field.
For sources whose extension is smaller than the domain size, the impact of the boundaries on the evolution of the solute distribution is similar to the one observed for the point sources discussed in Section 4.1. Thus, the results are not shown here.

We now investigate the impact of the source size on the temporal behavior of $D^{\text {ens }}(t), \bar{D}^{e}(t)$ and $\bar{D}^{a}(t)$.

For one given realization of the stratified medium the temporal evolution of particle distributions starting from the three sources under consideration are illustrated in Figs. 8 and 9. The extended sources were instantaneously and symmetrically injected about $y^{\prime}=0$. The snap shots of the spatial distribution are taken after $t=10^{-4} \tau_{\mathrm{D}}, t=10^{-2} \tau_{\mathrm{D}}, t=10^{-1} \tau_{\mathrm{D}}$ and $t=\tau_{\mathrm{D}}$.

At times of the order of the microscopic dispersion scale $\tau_{\mathrm{D}}^{\mathrm{m}}$, the small vertical extended sources experience the conductivity contrast along its vertical sampling extension. It is clear that the conductivity contrast experienced by the initial extended source of $100 l$ is greater than the one experienced by the $10 l$ source. This is illustrated in Figs. 8a and 9. With increasing time the concentration distributions becomes more spread out in the horizontal direction, see Figs. 8b and 9b. For the solute distribution for the line source of 1001 the memory of the source extension is wiped out with increasing time, even for times smaller than the time for complete vertical mixing. The solute distributions are practically identical for both source distributions already for $t=10^{-1} \tau_{\mathrm{D}}$, see Figs. $8 \mathrm{c}$ and $9 \mathrm{c}$. For times of the order of or larger than $\tau_{\mathrm{D}}$, the solute par-
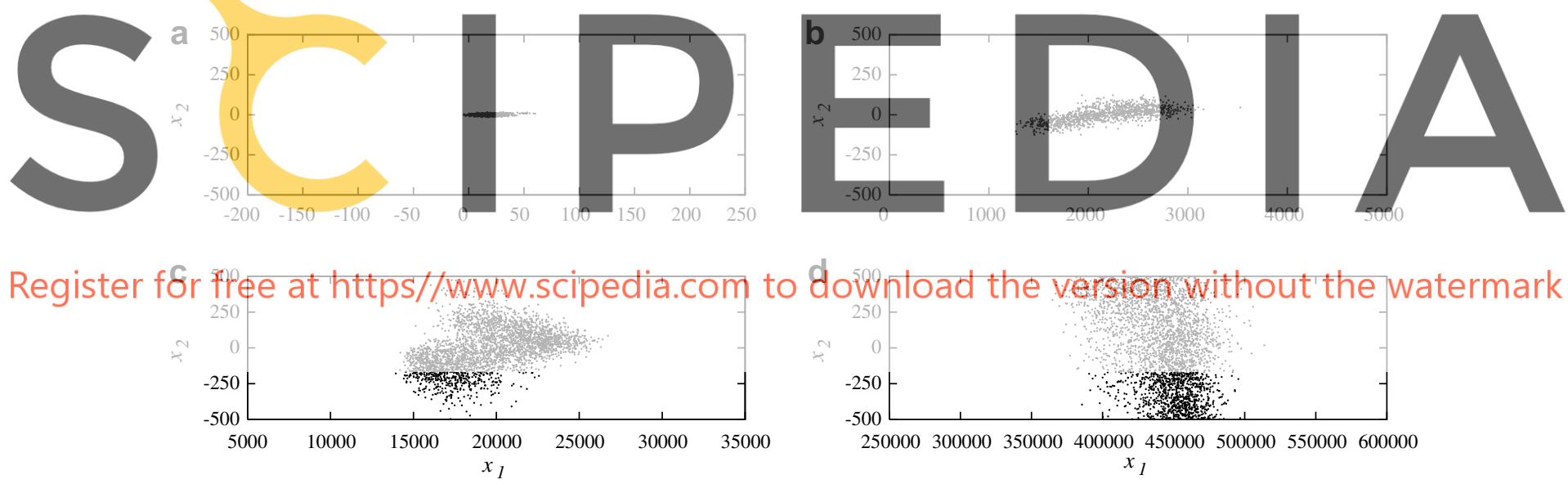

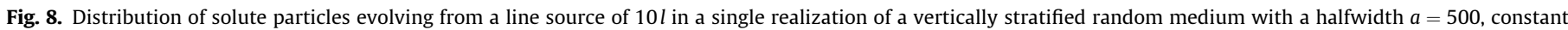
strata thickness $l=1$, local dispersion $D_{L}=D_{\mathrm{T}}=1$, after (a) $t=10^{-4} \tau_{\mathrm{D}}$, (b) $t=10^{-2} \tau_{\mathrm{D}}$, (c) $t=10^{-1} \tau_{\mathrm{D}}$, and (d) $t=\tau_{\mathrm{D}}$.
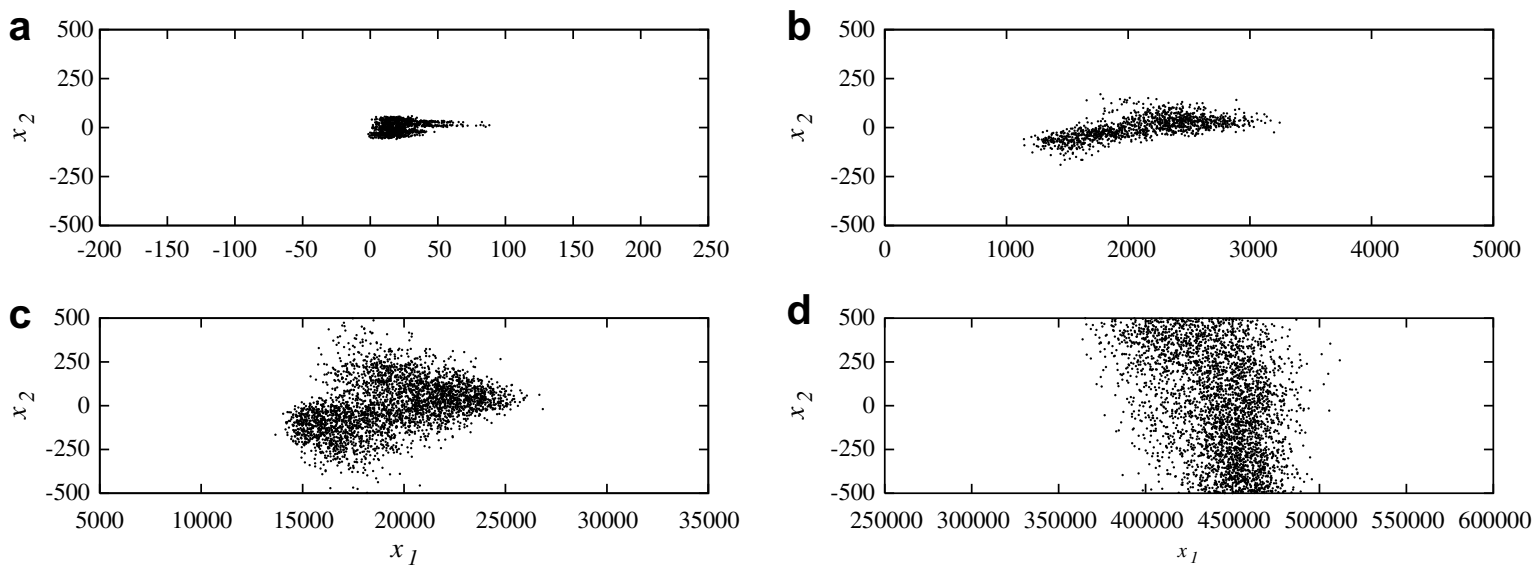

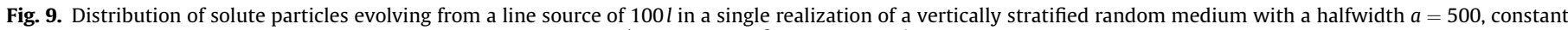
strata thickness $l=1$, local dispersion $D_{L}=D_{\mathrm{T}}=1$, after (a) $t=10^{-4} \tau_{\mathrm{D}}$, (b) $t=10^{-2} \tau_{\mathrm{D}}$, (c) $t=10^{-1} \tau_{\mathrm{D}}$, and (d) $t=\tau_{\mathrm{D}}$. 


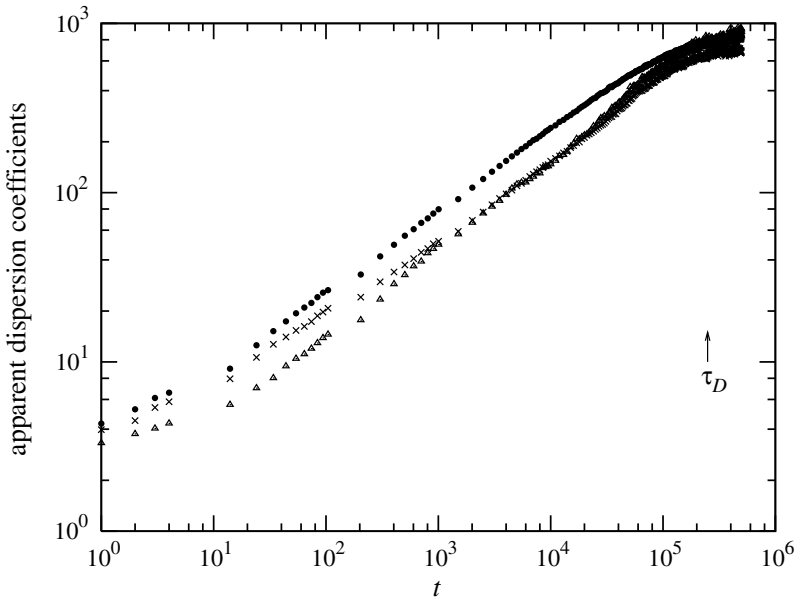

Fig. 10. Numerical results of the global apparent dispersion coefficients for extended sources of $10 l$ in triangles, $10^{2} l$ in crosses, and $10^{3} l$ in filled dots.

ticles are distributed uniformly over the medium cross-section in both cases, Figs. 8d and 9d.

The memory of the source size is reflected in the behavior of the apparent dispersion coefficient as outlined in the following. By definition, $\bar{D}^{a}(t)$ quantifies for pre-asymptotic times, purely advective spreading of the solute due to the velocity contrast within the initial source. The apparent dispersion coefficients for the extended sources of $10 \mathrm{l}$ and $10^{2} \mathrm{l}$ and $10^{3} \mathrm{l}$ are shown in Fig. 10. With increasing vertical extension of the initial source, $\bar{D}^{a}(t)$ evolves faster to the common asymptotic long time value. As observed in one given realization, the memory of the initial source size is lost for times smaller than the dispersion time scale, here $t \approx 10^{-2} \tau_{\mathrm{D}}$, and the apparent dispersion coefficients of the small extended sources are identical. Note that as shown in Figs. 11 and 12, when the memory of the initial source size is wiped out, the apparent and the global effective dispersion coefficients show the same temporal behavior.

As shown in Figs. 13 and 14 the global effective and the ensemble dispersion coefficients are not impacted in a sensible manner by the source size. For the three initial sources under consideration, $D^{\text {ens }}(t)$ and $\bar{D}^{e}(t)$ show a similar behavior which is controlled at long times by the dispersion time scale $\tau_{\mathrm{D}}$.

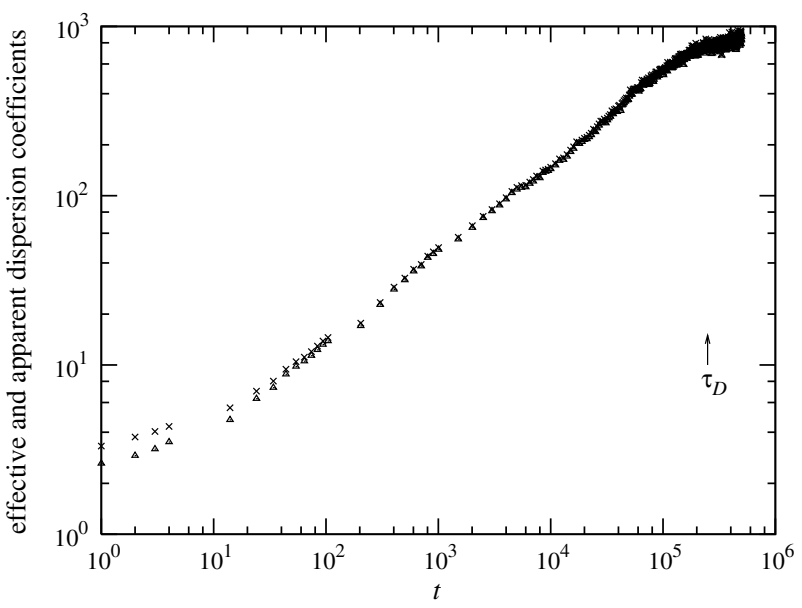

Fig. 11. Numerical results of the global effective and apparent dispersion coefficients for an extended source of $10 \mathrm{l}$.

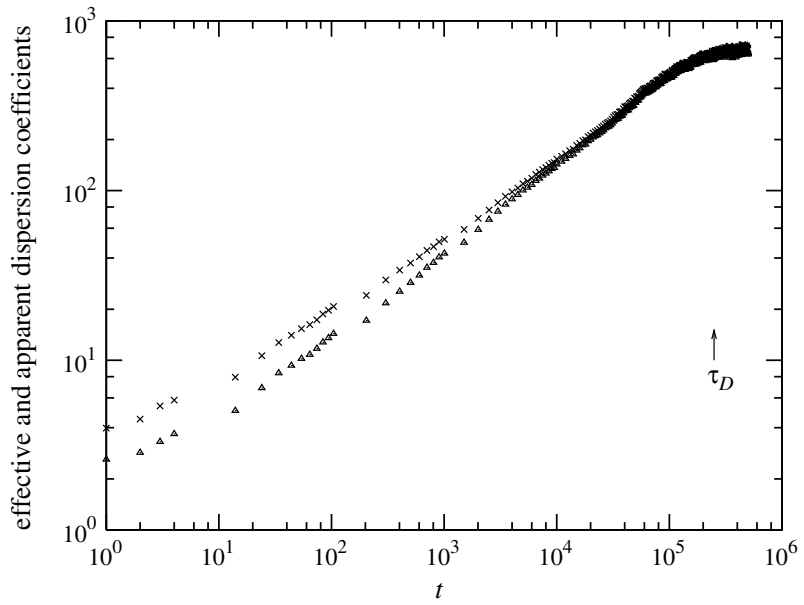

Fig. 12. Numerical results of the global effective (triangles) and apparent dispersion coefficients (crosses) for an extended source of $10^{2} l$.

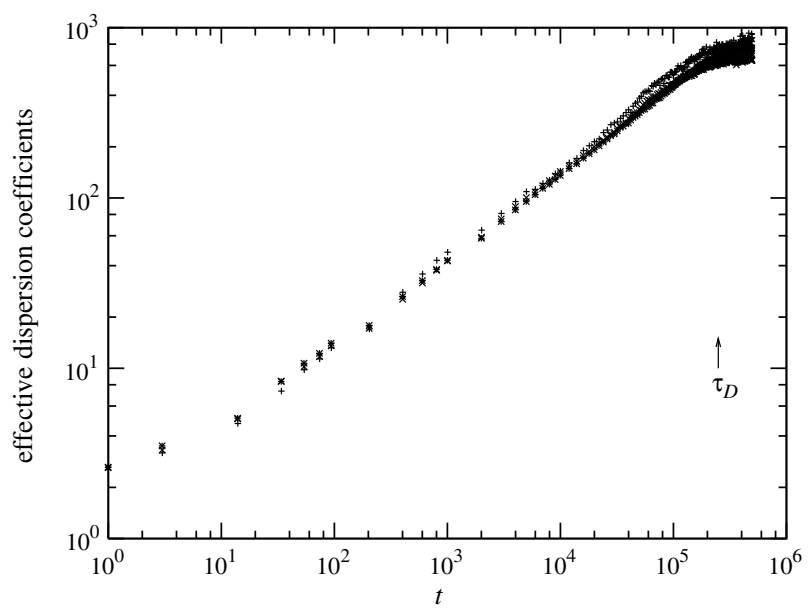

Fig. 13. Numerical results of the global effective dispersion coefficients for three extended sources of $10 l, 100 l$, and a line source that extends over the whole medium cross-section of $1000 \mathrm{l}$.

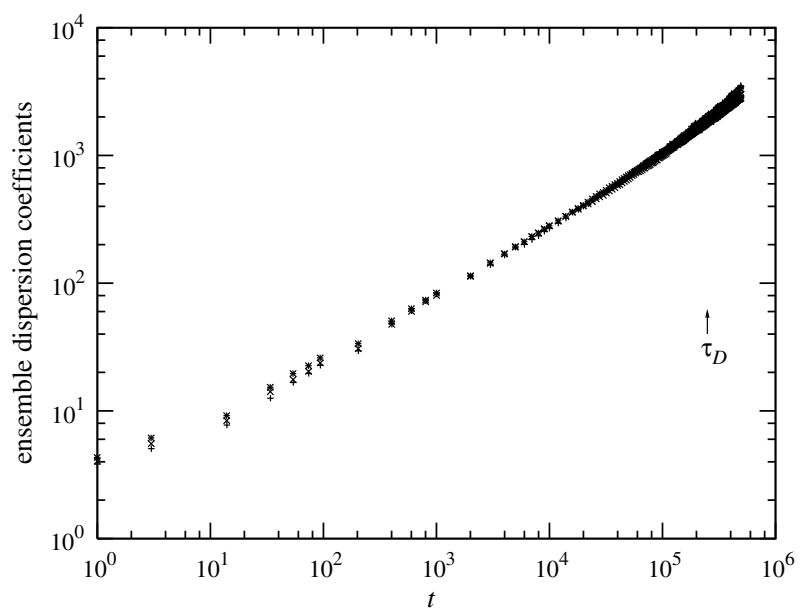

Fig. 14. Numerical results of the ensemble dispersion coefficients for three extended sources of $10 l, 100 l$, and a line source that extends over the whole medium cross-section of $1000 l$. 


\section{Summary and conclusions}

Even though highly idealized, stratified media present a valuable conceptual tool to investigate fundamental problems on transport in random media. The presented study analyzed preasymptotic non-Fickian transport features and the correct quantification of mixing and spreading by suitably defined effective dispersion coefficients. The correct quantification and representation of solute mixing is of importance for a series of applications as, e.g., bioremediation, natural attenuation, mixing limited chemical reaction, that is, whenever the efficiency of a chemical or biological process depends on the mixing of the participating species. We studied the quantification of mixing and spreading in confined media using stochastic modeling and quantified its limitations due to the intrinsic non-ergodicity of the confined media realizations. Memory effects on the effective solute spreading and mixing due source size and positions within the medium are quantified in terms of suitably defined (ensemble) dispersion coefficients, as summarized in the following.

For point-like initial distributions, the second centered moment of the concentration distribution (identical to the transport Green function), quantifies the interaction of vertical conductivity variations and local dispersion on the effective spreading and mixing properties of a solute. For an extended source this is different. For times smaller than the dispersion time for complete vertical solute mixing $\tau_{\mathrm{D}}=a^{2} / D$, the width of the solute distribution reflects purely advective spreading effects due to velocity fluctuations within the initial plume. Thus, following [24], for extended initial conditions solute spreading and mixing is quantified in terms of local distributions which originate from the point-sources that constitute the extended source. We thus define global effective dispersion coefficients as the weighted average over the local effective dispersion coefficients that characterize spreading and mixing for the solute distributions that evolve from the point sources that form the extended initial plume. It thus represents a global measure for the heterogeneity impact on mixing and spreading. Furthermore, we define the apparent dispersion coefficient as the half rate of change of the width of a distribution evolving from an extended initial plume. For early times, it reflects the purely advective spreading effects due to velocity variations in the extended initial distribution.

For the randomly stratified media under consideration here, the impact of vertical conductivity fluctuations on effective transport is quantified within a stochastic modeling framework. Effective and apparent dispersion coefficient are defined as ensemble averages of their single realization counterparts. In addition, we define a local ensemble dispersion coefficient, which is derived from the average transport Green function. The global ensemble dispersion coefficient for an extended initial plume then is given by the average of the local counterpart over the initial distribution. While the apparent dispersion coefficient quantifies center of mass fluctuations within the initial plume for a given realization, the ensemble dispersion coefficient quantifies the artificial spreading effect of center of mass fluctuations from realization to realization of the stratified random medium.

For an infinite stratified medium, the ensemble average leads to vertical homogenization and makes the average medium isotropic so that the effective and ensemble dispersion coefficients do not depend on the position of the source distribution. This symmetry is broken for the confined medium so that even in average, the memory of the initial position is preserved in the behavior of the effective and ensemble dispersion coefficients. As the medium is confined by impermeable horizontal boundaries (in the random walk picture the impermeable boundaries are modeled as reflecting walls), the vertical position of the initial injection affects the temporal behavior of the effective dispersion coefficients. For point sources located in the vicinity of the medium boundaries, at early times, the time needed for the solute to sample the entire medium cross-section is longer than for a solute that originates from an initial plume in the center of. Thus, $D^{e}\left(t \mid y^{\prime}\right)$ evolves slower for $y^{\prime}$ close to the boundaries than for $y^{\prime}$ located in the vicinity of the medium axis. On the other hand, as the concentration distribution remains longer near the boundaries, we have a bigger contrast between the center of mass velocities in different realizations and the ensemble mean center of mass velocity, which causes a faster increase of $D^{\text {ens }}\left(t \mid y^{\prime}\right)$. Due to the finite vertical extent of the medium, sample to sample fluctuations of the center of mass velocity persist and lead to a linear increase of the ensemble dispersion coefficient for $t>\tau_{\mathrm{D}}$.

Due to the finite number of strata in each realization, the model media cannot be ergodic. This is reflected in the variance of the spatial average of the conductivity fluctuations, which is inversely proportional to the lateral extent of the medium. This non-ergodicity is reflected in the behavior of the ensemble dispersion coefficient, which, at asymptotic times, increases linearly with time reflecting the finite size sample to sample fluctuations of the center of mass position. In the limit of infinite vertical extent, the center of mass fluctuations persist but their growth rate is weaker, as reflected by the difference between ensemble and effective dispersion coefficients.

For an initial plume that is spread over the whole medium cross-section, the global effective dispersion coefficient quantifies the average spreading and mixing behavior. It evolves slower than the apparent dispersion coefficient, which, at early times, reflects purely advective spreading due to local center of mass fluctuations within the extended initial distribution. At asymptotic times both coefficients converge to the Taylor dispersion coefficient. In the limit of infinite vertical extent (and thus also infinitely extended source distribution), the apparent and ensemble dispersion coefficients converge; the vertical (spatial) average and the ensemble average are equivalent in this limit.

The non-Fickian transport features observed at pre-asymptotic times together with the clear evidence of memory effects on spreading and mixing indicate that transport is non-Markovian. Thus, the effective mixing and spreading dynamics and thus the effective evolution of the solute plume cannot be quantified within a Fickian transport paradigm. An effective average transport description to model pre-asymptotic transport is in general nonMarkovian. This needs to taken into account when modeling effective transport in disordered media.

\section{Acknowledgements}

The financial support of ENRESA (Empresa Nacional de Residuos Radioactivos), the European Union IP FUNMIG (Contract No. 516514) and the MEC project MODEST (Project No. CGL-200505171) is gratefully acknowledged. V.Z. acknowledges the financial support of the European Commission and the Departament d'Universitats, Recerca i Societat de la Informació de la Generalitat de Catalunya. M.D. gratefully acknowledges the financial support of the program 'Ramon y Cajal' of the Spanish Ministry of Education and Science (MEC).

\section{Appendix A. Axial moment equations}

The advection dispersion equation for solute transport trough a stratified medium can be solved explicitly by the definition of axial moment equations. For technical convenience, we rewrite the local moments as (e.g. $[33,24])$

$\mu^{(n)}\left(t \mid \mathbf{x}^{\prime}\right)=\int_{\Omega} \mathrm{d} \mathbf{y} c_{n}\left(\mathbf{y}, t \mid \mathbf{x}^{\prime}\right)$, 
where we defined the local axial moments $c_{n}\left(\mathbf{y}, t \mid \mathbf{x}^{\prime}\right)$ by

$c_{n}\left(\mathbf{y}, t \mid \mathbf{x}^{\prime}\right)=\int_{-\infty}^{\infty} \mathrm{d} x_{1} x_{1}^{n} g\left(\mathbf{x}, t \mid \mathbf{x}^{\prime}, 0\right)$.

The $c_{0}\left(\mathbf{y}, t \mid \mathbf{x}^{\prime}\right)$ is the vertical concentration profile integrated over the $x_{1}$-direction of a solute that evolves from a point injection at $\mathbf{x}^{\prime}$ at time $t=0$. The local axial center of mass at the vertical position $\mathbf{y}$ at time $t$ is given by $c_{1}\left(\mathbf{y}, t \mid \mathbf{x}^{\prime}\right)$, while $c_{2}\left(\mathbf{y}, t \mid \mathbf{x}^{\prime}\right)$ is a measure for the width of the solute distribution at the vertical position $\mathbf{y}$ at time $t$.

By multiplication of (26) with $x_{1}^{n}$ and subsequent integration over $x_{1}$, we obtain for $n=0,1,2$ the transport equations (e.g. [24])

$\frac{\partial c_{0}\left(\mathbf{y}, t \mid \mathbf{y}^{\prime}\right)}{\partial t}-D_{\mathrm{T}} \nabla_{\mathbf{y}}^{2} c_{0}\left(\mathbf{y}, t \mid \mathbf{y}^{\prime}\right)=0$

$\frac{\partial c_{1}\left(\mathbf{y}, t \mid \mathbf{x}^{\prime}\right)}{\partial t}-D_{\mathrm{T}} \nabla_{\mathbf{y}}^{2} c_{1}\left(\mathbf{y}, t \mid \mathbf{x}^{\prime}\right)=[1-k(\mathbf{y})] c_{0}\left(\mathbf{y}, t \mid \mathbf{y}^{\prime}\right)$,

$\frac{\partial c_{2}\left(\mathbf{y}, t \mid \mathbf{x}^{\prime}\right)}{\partial t}-D_{\mathrm{T}} \nabla_{\mathbf{y}}^{2} c_{2}\left(\mathbf{y}, t \mid \mathbf{x}^{\prime}\right)=2[1-k(\mathbf{y})] c_{1}\left(\mathbf{y}, t \mid \mathbf{x}^{\prime}\right)$

$$
+2 D_{L} c_{0}\left(\mathbf{y}, t \mid \mathbf{y}^{\prime}\right),
$$

where $\nabla_{\mathbf{y}}$ denotes the nabla operator in $\Omega$. The initial conditions are given by

$c_{n}\left(\mathbf{y}, 0 \mid \mathbf{x}^{\prime}\right)=x_{1}^{\prime n} \delta\left(\mathbf{y}-\mathbf{y}^{\prime}\right)$,

where $n=0,1,2$. The boundary conditions are

$\left.\mathbf{n} \cdot \nabla_{\mathbf{y}} c_{n}\left(\mathbf{y}, t \mid \mathbf{x}^{\prime}\right)\right|_{\mathbf{y} \in \partial \Omega}=0$,

for $n=0,1,2$. The diffusion equation (A.3) is the projection of (26) from $\Omega^{d}$ onto $\Omega$. The initial and boundary conditions (A.6) and (A.7) imply, (i) $c_{0}\left(\mathbf{y}, t \mid \mathbf{x}^{\prime}\right) \equiv c_{0}\left(\mathbf{y}, t \mid \mathbf{y}^{\prime}\right)$, and (ii)

$\int_{\Omega} \mathrm{d} \mathbf{y} c_{0}\left(\mathbf{y}, t \mid \mathbf{y}^{\prime}\right)=1$.

The expressions for the local axial moments and local moments are identical to the ones given in [24]. For completeness, we give here a brief summary. Applying Duhamel's principle, the solutions of (A.4) and (A.5) can be written in terms of the auxiliary function $c_{0}\left(\mathbf{y}, t \mid \mathbf{y}^{\prime}\right)$

$$
\begin{aligned}
c_{1}\left(\mathbf{y}, t \mid \mathbf{x}^{\prime}\right)= & \left(x_{1}^{\prime}+t\right) c_{0}\left(\mathbf{y}, t \mid \mathbf{y}^{\prime}\right)-\int_{0}^{t} \mathrm{~d} t^{\prime} \int_{\Omega} \mathrm{d} \mathbf{y}^{\prime \prime} \\
& \times c_{0}\left(\mathbf{y}, t-t^{\prime} \mid \mathbf{y}^{\prime \prime}\right) k\left(\mathbf{y}^{\prime \prime}\right) c_{0}\left(\mathbf{y}^{\prime \prime}, t^{\prime} \mid \mathbf{y}^{\prime}\right), \\
c_{2}\left(\mathbf{y}, t \mid \mathbf{x}^{\prime}\right)= & \left(x_{1}^{\prime 2}+2 D_{L} t\right) c_{0}\left(\mathbf{y}, t \mid \mathbf{y}^{\prime}\right)+2 \int_{0}^{t} \mathrm{~d} t^{\prime} \int_{\Omega} \mathrm{d} \mathbf{y}^{\prime \prime} \\
& \times c_{0}\left(\mathbf{y}, t-t^{\prime} \mid \mathbf{y}^{\prime \prime}\right)\left[1-k\left(\mathbf{y}^{\prime \prime}\right)\right] c_{1}\left(\mathbf{y}^{\prime \prime}, t^{\prime} \mid \mathbf{x}^{\prime}\right),
\end{aligned}
$$

where we used the Markov property of the vertical diffusion process.

According to (A.1), we now obtain for the first and second local moments

$$
\begin{aligned}
\mu^{(1)}\left(t \mid \mathbf{y}^{\prime}\right)= & x_{1}^{\prime}+t-\int_{\Omega} \mathrm{d} \mathbf{y}^{\prime \prime} \int_{0}^{t} \mathrm{~d} t^{\prime} k\left(\mathbf{y}^{\prime \prime}\right) c_{0}\left(\mathbf{y}^{\prime \prime}, t^{\prime} \mid \mathbf{y}^{\prime}\right), \\
\mu^{(2)}\left(t \mid \mathbf{y}^{\prime}\right)= & \left(x_{1}^{\prime}+t\right)^{2}+2 D_{L} t-2\left(x_{1}^{\prime}+t\right) \int_{\Omega} \mathrm{d} \mathbf{y}^{\prime \prime} \\
& \times \int_{0}^{t} \mathrm{~d} t^{\prime} k\left(\mathbf{y}^{\prime \prime}\right) c_{0}\left(\mathbf{y}^{\prime \prime}, t^{\prime} \mid \mathbf{y}^{\prime}\right)+2 \int_{\Omega} \mathrm{d} \mathbf{y}^{\prime \prime} \\
& \times \int_{\Omega} \mathrm{d} \mathbf{y} \int_{0}^{t} \mathrm{~d} t^{\prime} \int_{0}^{t^{\prime}} \mathrm{d} t^{\prime \prime} k\left(\mathbf{y}^{\prime \prime}\right) k(\mathbf{y}) c_{0}\left(\mathbf{y}^{\prime \prime}, t^{\prime}-t^{\prime \prime} \mid \mathbf{y}\right) c_{0}\left(\mathbf{y}, t^{\prime \prime} \mid \mathbf{y}^{\prime}\right) .
\end{aligned}
$$

\section{Appendix B. Average dispersion coefficients for the infinite medium}

Here, we detail the development of the expressions for the local and global dispersion coefficients for the hypothetical case of an infinite stratified medium. Expressions (60) and (61) can be rewritten as

$$
\begin{aligned}
D^{\text {ens }}\left(t \mid y^{\prime}\right)= & D_{L}+\frac{1}{2} \Delta \kappa \sigma^{2} l t+\frac{2}{D_{\mathrm{T}} \pi^{2}} \sigma^{2} l \sum_{n=1}^{\infty} \frac{\Delta \kappa}{(n \Delta \kappa)^{2}} \\
& \times\left[1-\exp \left(-\frac{D_{\mathrm{T}} \pi^{2}(n \Delta \kappa)^{2}}{4} t\right)\right]+\frac{2}{3 D_{\mathrm{T}} \pi^{2}} \sigma^{2} l \\
& \times \sum_{n=1}^{\infty}(-1)^{n} \frac{\Delta \kappa}{(n \Delta \kappa)^{2}} \text { times }\left[\exp \left(-\frac{D_{\mathrm{T}} \pi^{2}(n \Delta \kappa)^{2}}{4} t\right)\right. \\
& \left.-\exp \left(-D_{\mathrm{T}} \pi^{2}(n \Delta \kappa)^{2} t\right)\right] \cos \left(\pi y^{\prime} n \Delta \kappa\right)
\end{aligned}
$$

and

$$
\begin{aligned}
\bar{D}^{e}\left(t \mid y^{\prime}\right)= & D^{\mathrm{ens}}\left(t \mid y^{\prime}\right)-\frac{2}{D_{\mathrm{T}} \pi^{2}} \sigma^{2} l \sum_{n=1}^{\infty} \frac{\Delta \kappa}{(n \Delta \kappa)^{2}} \\
& \times\left[\exp \left(-\frac{D_{\mathrm{T}} \pi^{2}(n \Delta \kappa)^{2}}{4} t\right)-\exp \left(-\frac{D_{\mathrm{T}} \pi^{2}(n \Delta \kappa)^{2}}{2} t\right)\right] \\
& -\frac{2}{D_{\mathrm{T}} \pi^{2}} \sigma^{2} l \sum_{n=1}^{\infty}(-1)^{n} \frac{\Delta \kappa}{(n \Delta \kappa)^{2}}\left[\exp \left(-\frac{D_{\mathrm{T}} \pi^{2}(n \Delta \kappa)^{2}}{4} t\right)\right. \\
& \left.-\exp \left(-\frac{D_{\mathrm{T}} \pi^{2}(n \Delta \kappa)^{2}}{2} t\right)\right] \cos \left(\pi y^{\prime} n \Delta \kappa\right),
\end{aligned}
$$

where we set $\tau_{\mathrm{D}}=a^{2} / D_{\mathrm{T}}$ and defined $\Delta \kappa \equiv 1 / a$. For $a \rightarrow \infty$, i.e., $\Delta \kappa \rightarrow 0$ we take the continuum limit. We define $n \Delta \kappa \equiv \kappa$ and substitute the summations in (B.1) and (B.2) by integrations over $\kappa$. This yields

$$
\begin{aligned}
D^{\mathrm{ens}}\left(t \mid y^{\prime}\right) \equiv & D^{\mathrm{ens}}(t) \\
= & D_{L}+\frac{2 \sigma^{2} l}{D_{\mathrm{T}} \pi} \int_{0}^{\infty} \frac{\mathrm{d} \kappa}{\kappa^{2}}\left[1-\exp \left(-\frac{D_{\mathrm{T}} \kappa^{2}}{4} t\right)\right], \\
\bar{D}^{e}\left(t \mid y^{\prime}\right) \equiv & \bar{D}^{e}(t) \\
= & D^{\mathrm{ens}}(t)-\frac{2 \sigma^{2} l}{D_{\mathrm{T}} \pi} \\
& \int_{0}^{\infty} \frac{\mathrm{d} \kappa}{\kappa^{2}}\left[\exp \left(-\frac{D_{\mathrm{T}} \kappa^{2}}{4} t\right)-\exp \left(-\frac{D_{\mathrm{T}} \kappa^{2}}{2} t\right)\right] .
\end{aligned}
$$

Note that the linear term in (B.1) as well as the terms containing the initial position $y^{\prime}$ in (B.1) and (B.2) cancel out in this limit. The integrals in (B.3) and (B.4) can be performed straightforwardly and give (66) and (67), respectively.

\section{References}

[1] Matheron M, de Marsily G. Is the transport in porous media always diffusive? A counter-example. Water Resour Res 1980;16:901-17.

[2] Güven O, Molz FJ, Melville JG. An analysis of macrodispersion in a stratified aquifer. Water Resour Res 1984;20(10):1337-53.

[3] Marle C, Simandoux P, Pacsirsky J, Gaulier C. Etude du deplacement de fludes miscibles en milieu poreux stratifie. Rev Inst Fr Pet 1967;22:272-94.

[4] Güven O, Molz FJ. Deterministic and stochastic analyses of dispersion in an unbounded stratified porous medium. Water Resour Res 1986;22(11): $1556-74$

[5] Gupta VK, Bhattacharya RN. Solute dispersion in multidimensional periodic saturated porous media. Water Resour Res 1986;22(2):156-64.

[6] Dagan G. Transport in heterogeneous porous formations: spatial moments, ergodicity, and effective dispersion. Water Resour Res 1990;26(6):1281-90.

[7] Salandin P, Rinaldo A, Dagan G. A note on transport in stratified formations by flow tilted with respect to the beding. Water Resour Res 1991;27:3009-17. 
[8] Rajaram H, Gelhar L. Plume scale dependent dispersion in heterogeneous aquifers 1. Lagrangian analysis in stratified aquifer. Water Resour Res 1993;29:3249-60.

[9] Sposito G, Weeks SW. Tracer advection by steady groundwater flow in a stratified aquifer. Water Resour Res 1998:34:1051-9.

[10] Clincy M, Kinzelbach H. Stratified disordered media: exact solutions for transport parameters and their self-averaging properties. J Phys A: Math Gen 2001;34:7141-52.

[11] Fiori A, Dagan G. Transport of a passive scalar in a stratified porous medium. Transp Porous Media 2002;47:81-98.

[12] Le Borgne T, de Dreuzy JR, Davy O, Bour. Characterization of the velocity field organization in heterogeneous media by conditional correlation. Water Resour Res 2007;43:W02419.

[13] Dentz M, Le Borgne T, Carrera J. Effective transport in random shear flows. Phys Rev E 2008;77:020101(R).

[14] Bouchaud J, Georges A. Anomalous diffusion in disordered media: statistical mechanisms, models and physical applications. Phys Rep 1990;195:127-293.

[15] Bouchaud J, Georges A, Koplik J, Provata A, Redner S. Superdiffusion in random velocity fields. Phys Rev Lett 1990;64:2503-6.

[16] Compte A, Caceres M. Fractional dynamics in random velocity fields. Phys Rev Lett 1998;81:3140-3.

[17] Majumdar SN. Persistence of a particle in the Matheron-de Marsily velocity field. Phys Rev E 2003;68:050101R.

[18] Young WR, Jones S. Shear dispersion. Phys Fluids A 1991;3(5):1087-101.

[19] Camacho J. Purely global model for Taylor dispersion. Phys Rev E 1993:48(1):310-20.

[20] Berensten CWJ, Verlaan ML, van Kruijsdijk CPJW. Upscaling and reversibility of Taylor dispersion in heterogeneous porous media. Phys Rev E 2005;71:046308.
[21] Berensten CWJ, Verlaan ML, van Kruijsdijk CPJW. Upscaling, relaxation and reversibility of dispersive flow in stratified porous media. Transp Porous Media 2006;68(2):187-218.

[22] Taylor GI. Dispersion of soluble matter in solvent flowing through a tube. Proc Roy Soc A 1953;219:186-203.

[23] Kitanidis PK. The concept of the dilution index. Water Resour Res 1994;30:2011-26

[24] Dentz M, Carrera J. Mixing and spreading in stratified flow. Phys Fluids 2007;19(1):017107.

[25] Kitanidis PK. Prediction by the method of moments of transport in a heterogeneous formation. J Hydrol 1988;102:453-73.

[26] Dentz M, Kinzelbach $H$, Attinger S, Kinzelbach W. Temporal behavior of solute cloud in a heterogeneous porous medium: 1. Point-like injection. Water Resour Res 2000;36(12):3591-604.

27] Bear J. Dynamics of fluids in porous media. American Elsevier; 1972.

[28] Dentz M, Berkowitz B. Exact effective transport dynamics in a onedimensional random environment. Phys Rev E 2005;72(3):031110.

[29] Abramowitz M, Stegun IA. Handbook of mathematical functions. Dover Publications; 1972.

[30] Kitanidis PK. Statistical estimation of polynomial generalized covariance functions and hydrologic applications. Water Resour Res 1983;19(4): 909-21.

[31] Dentz M, Carrera J. Effective solute transport in temporally fluctuating flow through heterogeneous media. Water Resour Res 2005;41:W08414.

[32] Risken H. The Fokker-Planck equation. Heidelberg, New York: Springer; 1996.

[33] Aris R. On the dispersion of a solute in a fluid flowing through a tube. Proc Roy Soc Lond A 1956;235:67-77. 